\title{
Alum-Catalyzed One-Pot Synthesis of Dihydropyrano[3,2-b]chromenediones
}

\author{
Wei Lin Li, Li Qiang Wu* and Fu Lin Yan \\ School of Pharmacy, Xinxiang Medical University, Xinxiang, Henan 453003, P. R. China
}

\begin{abstract}
Neste trabalho é descrito um procedimento livre de solventes, limpo, simples e eficiente para a síntese de diidropirano[3,2- $b$ ]cromenodionas (onze derivados) a partir da reação de condensação de três componentes de ácido kójico, aldeídos aromáticos e dimedona, utilizando alume como catalisador reutilizável.
\end{abstract}

A simple, green and efficient solventless procedure for the synthesis of eleven dihydropyrano[3,2-b]chromenediones from the three-component condensation reaction of kojic acid, aromatic aldehydes, and dimedone using a reusable alum as catalyst is described.

Keywords: dihydropyrano[3,2-b]chromenedione, alum, kojic acid, solvent-free reaction

\section{Introduction}

The rapid assembly of molecular diversity is an important goal of synthetic organic chemistry and one of the key paradigms of modern drug discovery. One approach to address this challenge involves the development of multicomponent reactions (MCR), in which three or more reactants are combined together in a single reaction flask to generate a product. In addition to the intrinsic atom economy and selectivity underlying such reactions, simpler procedures and equipment, time and energy savings, as well as environmental friendliness have all led to a sizable effort to design and implement MCR in both academia and industry. ${ }^{1}$

Kojic acid derivatives, one of the important kind of fused heterocyclic compounds, possess significant bioactivities such as antifungal, ${ }^{2}$ anti-neoplastic, ${ }^{3}$ antiproliferative, ${ }^{4}$ anti$\mathrm{HIV},{ }^{5}$ anticonvulsant, ${ }^{6}$ anti-inflammatory, ${ }^{7}$ antioxidative, ${ }^{8}$ antibacterial, ${ }^{9}$ anticonvulsant, ${ }^{10}$ and tyrosinase inhibitory activities. ${ }^{11}$ Therefore, the synthesis of kojic acid derivatives has aroused great interest in the organic and medicinal communities.

Recently Reddy et al. ${ }^{12}$ reported the synthesis of 2-(hydroxymethyl)-7,7-dimethyl-10-aryl-7,8-dihydropyrano[3,2-b]chromene-4,9(6H,10H)-diones using $\mathrm{InCl}_{3}$ as a catalyst. However, this method is associated with one or more disadvantages such as the use of an expensive, waterintolerant and non recyclable catalyst and higher reaction temperature. Thus, there is still need of a simple and general

*e-mail: wliq870@163.com procedure for the one-pot synthesis of 2-(hydroxymethyl)7,7-dimethyl-10-aryl-7,8-dihydropyrano[3,2- $b]$ chromene$4,9(6 H, 10 H)$-dione under mild conditions.

Alum $\left(\mathrm{KAl}\left(\mathrm{SO}_{4}\right)_{2} \cdot 12 \mathrm{H}_{2} \mathrm{O}\right)$, which is relatively nontoxic and inexpensive catalyst, has emerged as an efficient alternative catalyst for a variety of prominent organic transformations, such as the Biginelly ${ }^{13}$ and Pechmann ${ }^{14}$ reactions and also used for the synthesis of 1,8-dioxo-octahydroxanthenes,,$^{15}$ isoquinolonic acids, ${ }^{16}$ trisubstituted imidazoles, ${ }^{17} 1^{\prime}$ ' $H$-spiro[isoindoline-1,2'quinazoline]-3,4' (3' $H$ )-diones, ${ }^{18}$ 1,3,4-oxadiazoles ${ }^{19}$ and 1,5-benzodiazepines. ${ }^{20}$ In this paper, we wish to report a rapid and highly efficient method for the synthesis of 2-(hydroxymethyl)-7,7-dimethyl-10-aryl-7,8dihydropyrano[3,2-b] chromene-4,9(6H,10H)-dione in the presence of alum under solvent-free conditions (Scheme 1).

\section{Results and Discussion}

To choose optimum conditions, first, the effect of temperature on the rate of the reaction was studied for the preparation of the three-component condensation reaction of kojic acid, benzaldehyde, and dimedone in presence of $10 \mathrm{~mol} \%$ alum under solvent-free conditions (Table 1). At $100{ }^{\circ} \mathrm{C}$, the reaction proceeded smoothly and gave short reaction time and high yield. Therefore, we kept the reaction temperature at $100{ }^{\circ} \mathrm{C}$.

Next, in the study set out to determine optimal amount of alum, the reaction was carried out by varying the amount of the catalyst (Table 2). Maximum yield was obtained with $10 \mathrm{~mol} \%$ of the catalyst. Further increase in amount of alum 
<smiles>CC1(C)CC(=O)CC(C)(C)C1</smiles>

Scheme 1.

Table 1. Temperature optimization for the synthesis of 2-(hydroxymethyl)7,7-dimethyl-10-phenyl-7,8-dihydropyrano[3,2-b]chromene$4,9(6 H, 10 H)$-dione $^{\mathrm{a}}$

\begin{tabular}{lccc}
\hline entry & $\mathrm{T} /{ }^{\circ} \mathrm{C}$ & time $/$ min & yield $/ \%^{\mathrm{b}}$ \\
\hline 1 & 25 & 240 & 0 \\
2 & 50 & 120 & $<5$ \\
3 & 60 & 120 & 12 \\
4 & 70 & 90 & 38 \\
5 & 80 & 90 & 53 \\
6 & 90 & 60 & 76 \\
7 & 100 & 50 & 92 \\
8 & 110 & 50 & 92 \\
9 & 120 & 40 & 90 \\
10 & 130 & 40 & 92 \\
11 & 140 & 40 & 91 \\
\hline
\end{tabular}

aReaction conditions: kojic acid $(1 \mathrm{mmol})$; benzaldehyde $(1 \mathrm{mmol})$; dimedone ( $1 \mathrm{mmol})$; alum $(0.1 \mathrm{mmol})$; without solvent. ${ }^{\mathrm{b}}$ Isolated yield.

in the mentioned reaction did not has any significant effect on the product yield.

In order to extend the above reaction (Scheme 1) to a library system, various kinds of arylaldehydes $\mathbf{2}$ (Table 3) were subjected to react with $\mathbf{1}$ and $\mathbf{3}$ to give the
Table 2. The amounts of catalyst optimization for the synthesis of 2-(hydroxymethyl)-7,7-dimethyl-10-phenyl-7,8-dihydropyrano[3,2-b] chromene-4,9(6H,10H)-dione ${ }^{\mathrm{a}}$

\begin{tabular}{lccc}
\hline entry & alum / mol\% & time / min & yield / \% \\
\hline 1 & 0 & 240 & 0 \\
2 & 1 & 180 & 49 \\
3 & 2 & 120 & 61 \\
4 & 5 & 90 & 76 \\
5 & 10 & 50 & 92 \\
6 & 15 & 50 & 89 \\
7 & 20 & 50 & 92 \\
\hline
\end{tabular}

${ }^{\mathrm{a}}$ Reaction conditions: kojic acid $(1 \mathrm{mmol})$; benzaldehyde $(1 \mathrm{mmol})$; dimedone $(1 \mathrm{mmol}) ; 100^{\circ} \mathrm{C}$; without solvent. ${ }^{\mathrm{b}}$ Isolated yield.

corresponding 2-(hydroxymethyl)-7,7-dimethyl-10-aryl7,8-dihydropyrano[3,2-b]chromene-4,9(6H,10H)-dione, and representative examples are shown in Table 3. All of 1 gave the expected products in high yields, either bearing electron-withdrawing groups (such as halide, nitro) or electron-donating groups (such as alkyl group) under the same reaction condition. To further demonstrate the scope and limitation of the substrates, aliphatic aldehydes, such as phenylacetaldehyde, propionaldehyde, $n$-butylaldehyde and

Table 3. Preparation of 2-(hydroxymethyl)-7,7-dimethyl-10-aryl-7,8-dihydropyrano[3,2-b]chromene-4,9(6H,10H)-dione a

\begin{tabular}{lccccc}
\hline entry & $\mathrm{Ar}$ & time $/ \mathrm{min}$ & product & yield $/ \%{ }^{\mathrm{b}}$ & $\mathrm{mp} /{ }^{\circ} \mathrm{C}($ lit. $)$ \\
\hline 1 & $\mathrm{C}_{6} \mathrm{H}_{5}$ & 50 & $\mathbf{4 a}$ & $92(90,86)^{\mathrm{c}}$ & $184-185(186-188)^{12}$ \\
2 & $4-\mathrm{Cl}_{-} \mathrm{C}_{6} \mathrm{H}_{4}$ & 50 & $\mathbf{4 b}$ & 94 & $205-206$ \\
3 & $4-\mathrm{F}_{6} \mathrm{C}_{6} \mathrm{H}_{4}$ & 50 & $\mathbf{4 c}$ & 92 & $161-162(160-164)^{12}$ \\
4 & $4-\mathrm{NO}_{2}-\mathrm{C}_{6} \mathrm{H}_{4}$ & 40 & $\mathbf{4 d}$ & 91 & $229-230$ \\
5 & $3-\mathrm{NO}_{2}-\mathrm{C}_{6} \mathrm{H}_{4}$ & 45 & $\mathbf{4 e}$ & 95 & $212-213$ \\
6 & $2-\mathrm{Cl}_{-}-\mathrm{C}_{6} \mathrm{H}_{4}$ & 50 & $\mathbf{4 f}$ & 89 & $216-217$ \\
7 & $2,4-\mathrm{Cl}_{2}-\mathrm{C}_{6} \mathrm{H}_{3}$ & 50 & $\mathbf{4 g}$ & 91 & $166-167$ \\
8 & $3,4,5-(\mathrm{MeO})_{3}-\mathrm{C}_{6} \mathrm{H}_{2}$ & 60 & $\mathbf{4 h}$ & 88 & $174-175$ \\
10 & $2,5-(\mathrm{MeO})_{2}-\mathrm{C}_{6} \mathrm{H}_{3}$ & 60 & $\mathbf{4 i}$ & 85 & $192-193$ \\
11 & $4-\mathrm{Me}-\mathrm{C}_{6} \mathrm{H}_{4}$ & 60 & $\mathbf{4 j}$ & 89 & 87 \\
\hline
\end{tabular}

aReaction conditions: kojic acid $(1 \mathrm{mmol})$; aldehyde $(1 \mathrm{mmol})$; dimedone $(1 \mathrm{mmol})$; alum $(0.1 \mathrm{mmol})$; without solvent; $100^{\circ} \mathrm{C}$. ${ }^{\mathrm{b}}$ Isolated yield. ${ }^{\mathrm{c}}$ The catalyst was reused for three runs. 
<smiles>[R]C=C1C(=O)CC(C)(C)CC1=O</smiles><smiles>[R]C1C2=C(CC(C)(C)CC2=O)Oc2oc(CO)cc(=O)c21</smiles>

Scheme 2.

$n$-heptaldehyde, were used as reactants to react with kojic acid and dimedone. However, the desired products were not found and obtained successfully. All of the structures were characterized by IR, ${ }^{1} \mathrm{H}$ NMR,,${ }^{13} \mathrm{C}$ NMR, MS and elemental analysis. In the ${ }^{1} \mathrm{H}$ NMR spectra, there was a missing proton, which was possibly $\mathrm{OH}$. When ${ }^{1} \mathrm{H}$ NMR spectra were determined using DMSO- $d_{6}$ instead of $\mathrm{CDCl}_{3}$ as solvent, there appeared a broad peak at $\delta 5.54-5.36$, so that proved that our supposition was correct.

We also checked the reusability of the catalyst by recovering the alum and using it for new runs and found that the catalyst could be reused several times without any decrease in the product yield. An example is shown for the reaction of kojic acid with benzaldehyde (Table 3, 4a) and dimedone. The catalyst can be removed from the aqueous phase by removing the water under vacuum then washing with EtOH and drying at rt.

The formation of products $4 \mathbf{a}-\mathbf{4 k}$ can be rationalized by initial formation of heterodiene $\mathbf{5}$ by standard Knoevenagel condensation of dimedone $\mathbf{3}$ and aromatic aldehyde $\mathbf{2}$ in the presence of a catalytic amount of alum. Subsequent Diels Alder-type addition of kojic acid $\mathbf{1}$ to the heterodienes $\mathbf{5}$ followed by dehydration afford the corresponding products 4a-4k (Scheme 2).

\section{Conclusions}

In conclusion, an efficient protocol for the one-pot preparation of 2-(hydroxymethyl)-7,7-dimethyl-10-aryl7,8-dihydropyrano[3,2-b]chromene-4,9(6H,10H)-diones from the three-component condensation reaction of kojic acid, aromatic aldehydes, and dimedone employing alum as reusable catalyst was described. The reactions were carried out under thermal solvent-free in short reaction time and produced the corresponding products in good to excellent yields. Also, the catalyst could be successfully recovered and recycled at least for three runs without significant loss in activity.

\section{Supplementary Information}

Experimental details and spectroscopic data are available free of charge at http://jbcs.sbq.org.br as PDF file.

\section{Acknowledgments}

We are pleased to acknowledge the financial support from Xinxiang Medical University.

\section{References}

1. Bienayme, H.; Hulme, C.; Oddon, G.; Schmitt, P.; Chem. Eur. J. 2000, 6, 3321.

2. Balaz, S.; Uher, M.; Brtko, J.; Veverka, M.; Bransova, J.; Dobias, J.; Podova, M.; Buchvald, J.; Folia microbiol. 1993, 38, 387; Uher, M.; Cizmarik, J.; Farm. Obz. 2001, 70, 46.

3. Novotny, L.; Rauko, P.; Abdel-Hamid, M.; Vachalkova, A.; Neoplasma 1999, 46, 89.

4. Fickova, M.; Pravdova, E.; Rondhal, L.; Uher, M.; Brtko, J.; J. Appl. Toxicol. 2008, 28, 554.

5. Tanaka, R.; Tsujii, H.; Yamada, T.; Kajimoto, T.; Amano, F.; Hasegawa, J.; Hamashima, Y.; Node, M.; Katoh, K.; Takebe, Y.; Bioorg. Med. Chem. 2009, 17, 5238. 
6. Aytemir, M. D.; Septioglu, E.; Calis, U.; Arzneim. Forsch. 2010, $60,22$.

7. Rho, H. S.; Ahn, S. M.; Yoo, D. S.; Kim, M. K.; Cho, D. H.; Cho, J. Y.; Bioorg. Med. Chem. Lett. 2010, 20, 6569.

8. Abe, Y.; Takahashi, Y.; Yukagaku 1970, 19, 23.

9. Kotani, T.; Ichimoto, I.; Tatsumi, C.; Hakko Kogaku Zasshi 1973, 51, 66.

10. Kim, J.; Lim, S.; Yakhak Hoechi 1999, 43, 28.

11. Aytemir, M. D.; Septioglu, E.; Calis, U.; Arzneim. Forsch. 2010, 60, 22.

12. Subba Reddy, B. V.; Ramana Reddy, M.; Narasimhulu, G.; Yadav, J. S.; Tetrahedron Lett. 2010, 51, 5677.

13. Azizian, J.; Mohammadi,A.A.; Karimi, A. R.; Mohammadizadeh, M. R.; Appl. Catal., A 2006, 300, 85.

14. Dabiri, M.; Baghbanzadeh, M.; Kiani, S.; Vakilzadeh, Y.; Monatsh. Chem. 2007, 138, 997.
15. Madje, B. R.; Ubale, M. B.; Bharad, J. V.; Shingare, M. S.; S. Afr. J. Chem. 2009, 63, 36.

16. Azizian, J.; Mohammadi,A.A.; Karimi,A. R.; Mohammadizadeh, M. R.; J. Org. Chem. 2006, 71, 350.

17. Mohammadi, A. A.; Mivechi, M.; Kefayati, H.; Monatsh. Chem. 2008, 139, 935.

18. Mohammadi, A. A.; Qaraat, H.; Monatsh. Chem. 2009, 140, 401.

19. Dabiri, M.; Salehi, P.; Otokesh, S.; Baghbanzadeh, M.; Bahramnejad, M.; Monatsh. Chem. 2007, 38, 253.

20. Mahajan, D.; Naqvi, T.; Sharma, R. L.; Kapoor, K. K.; Aust. J. Chem. 2008, 61, 59.

Submitted: May 25, 2011

Published online: October 6, 2011 


\title{
Alum-Catalyzed One-Pot Synthesis of Dihydropyrano[3,2-b]chromenediones
}

\author{
Wei Lin Li, Li Qiang Wu* and Fu Lin Yan \\ School of Pharmacy, Xinxiang Medical University, Xinxiang, Henan 453003, P. R. China
}

\section{Experimental}

IR spectra were determined on an FTS-40 infrared spectrometer; NMR spectra were determined on Bruker $\mathrm{AV}-400$ spectrometer at room temperature using TMS as internal standard, coupling constants $(J)$ were measured in Hz. Mass spectra were recorded on a Finnigan LCQ Advantage mass spectrometer. Elemental analyses were performed by a Vario-III elemental analyzer. Melting points were determined on a XT-4 binocular microscope and were uncorrected. Commercially available reagents were used throughout without further purification unless otherwise stated.

General procedure for the preparation of 2-(hydroxymethyl)7,7-dimethyl-10-aryl-7,8-dihydropyrano[3,2-b]chromene4,9(6H,10H)-diones

A mixture of kojic acid (1 mmol), aldehyde (1 mmol), dimedone $(1 \mathrm{mmol})$ and alum $(0.1 \mathrm{mmol})$ was heated at $100{ }^{\circ} \mathrm{C}$ for an appropriate time (TLC). After completion of the reaction, the mixture was washed with water $(15 \mathrm{~mL})$ and the crude product was purified by column chromatography over silica gel using ethyl acetate-hexane (7:3) as eluent. The portions were combined and solvent evaporated under reduced pressure to provide compounds $\mathbf{4 a}-\mathbf{4 k}$ as solids. The solids were recrystallized from $\mathrm{EtOH}$ to give pure $\mathbf{4 a - 4 k}$ as white crystals. Aqueous washings were collected and evaporated under reduced pressure. After removal of the water, alum was recovered.

2-(Hydroxymethyl)-7,7-dimethyl-10-phenyl-7,8-dihydropyrano[3,2-b]chromene-4,9(6H,10H)-dione (4a)

$\operatorname{IR}(\mathrm{KBr}) v_{\max } / \mathrm{cm}^{-1}: 3362,3080,2952,2890,1667,1637$, 1441, 1378, 1219, 1193, 1078, 990, 950, 712; ${ }^{1} \mathrm{H}$ NMR $\left(\mathrm{CDCl}_{3}, 400 \mathrm{MHz}\right) \delta$ 7.32-7.21 (m, 5H), $6.50(\mathrm{~s}, 1 \mathrm{H}), 4.87$ (s, 1H), 4.43-4.32 (m, 2H), 2.71-2.59 (m, 2H), 2.29-2.19 $(\mathrm{m}, 2 \mathrm{H}), 1.11$ (s, 3H), $1.04(\mathrm{~s}, 3 \mathrm{H}) ;{ }^{13} \mathrm{C}$ NMR $\left(\mathrm{CDCl}_{3}\right.$, $100 \mathrm{MHz}) \delta$ 196.2, 171.3, 167.3, 163.9, 151.7, 140.5, 137.5,

*e-mail: wliq870@163.com
$128.8,128.1,127.8,112.3,112.2,60.6,50.4,40.9,38.4$, 32.3, 29.0, 27.4. MS (ESI): $m / z 353[\mathrm{M}+\mathrm{H}]^{+}$. Anal. Calc. for $\mathrm{C}_{21} \mathrm{H}_{20} \mathrm{O}_{5}$ : C 71.58, $\mathrm{H}$ 5.72; found: C 71.65, H 5.68.

2-(Hydroxymethyl)-7,7-dimethyl-10-(4-chlorophenyl)-7,8dihydropyrano $[3,2-b]$ chromene-4,9(6H,10H)-dione $(\mathbf{4 b})$

IR (KBr) $v_{\text {max }} / \mathrm{cm}^{-1}: 3325,2961,2930,2870,1672$, 1640, 1600, 1490, 1442, 1377, 1218, 1190, 1076, 1014, 952, 850; ${ }^{1} \mathrm{H} \mathrm{NMR}\left(\mathrm{CDCl}_{3}, 400 \mathrm{MHz}\right) \delta$ 7.28-7.18 (m, $4 \mathrm{H}), 6.52(\mathrm{~s}, 1 \mathrm{H}), 4.87(\mathrm{~s}, 1 \mathrm{H}), 4.41-4.37(\mathrm{~m}, 2 \mathrm{H}), 2.66-$ $2.60(\mathrm{~m}, 2 \mathrm{H}), 2.30-2.21(\mathrm{~m}, 2 \mathrm{H}), 1.11(\mathrm{~s}, 3 \mathrm{H}), 1.03(\mathrm{~s}, 3 \mathrm{H})$; ${ }^{13} \mathrm{C} \mathrm{NMR}\left(\mathrm{CDCl}_{3}, 100 \mathrm{MHz}\right) \delta 196.3,171.4,168.3,164.1$, 151.2, 138.9, 137.5, 133.6, 129.4, 129.0, 112.0, 111.9, 60.4, 50.3, 40.8, 37.8, 32.3, 28.9, 27.4. MS (ESI): $\mathrm{m} / z 387$ $[\mathrm{M}+\mathrm{H}]^{+}$. Anal. calc. for $\mathrm{C}_{21} \mathrm{H}_{19} \mathrm{ClO}_{5}: \mathrm{C} 65.20, \mathrm{H} 4.95$; found: C 65.12, H 4.88 .

2-(Hydroxymethyl)-7,7-dimethyl-10-(4-florophenyl)-7,8dihydropyrano[3,2-b]chromene-4,9(6H,10H)-dione (4c)

IR (KBr) $v_{\max } / \mathrm{cm}^{-1}: 3363,2953,2930,2853,1675$, 1638, 1601, 1509, 1443, 1378, 1219, 1192, 1157, 1075, 962, 845, 683; ${ }^{1} \mathrm{H}$ NMR $\left(\mathrm{CDCl}_{3}, 400 \mathrm{MHz}\right) \delta$ 7.24-7.20 (m, 2H), 6.99-6.95(m, 2H), $6.52(\mathrm{~s}, 1 \mathrm{H}), 4.87(\mathrm{~s}, 1 \mathrm{H})$, 4.40-4.36 (m, 2H), 2.65-2.61 (m, 2H), 2.25-2.22 (m, 2H), $1.10(\mathrm{~s}, 3 \mathrm{H}), 1.03(\mathrm{~s}, 3 \mathrm{H}) \cdot{ }^{13} \mathrm{C} \mathrm{NMR}\left(\mathrm{CDCl}_{3}, 100 \mathrm{MHz}\right)$ $\delta$ 196.4, 171.5, 168.3, 164.0, 160.9, 151.5, 137.4, 136.2, 129.7, 129.6, 115.8, 115.6, 112.2, 112.0, 60.4, 50.3, 40.8, 37.7, 32.3, 28.9, 27.4; MS (ESI): $\mathrm{m} / z 371[\mathrm{M}+\mathrm{H}]^{+}$. Anal. calc. for $\mathrm{C}_{21} \mathrm{H}_{19} \mathrm{FO}_{5}: \mathrm{C} 68.10, \mathrm{H} \mathrm{5.17}$; found: $\mathrm{C} 68.20$, H 5.12.

2-(Hydroxymethyl)-7,7-dimethyl-10-(4-nitrophenyl)-7,8dihydropyrano[3,2-b]chromene-4,9(6H,10H)-dione (4d)

IR (KBr) $v_{\text {max }} / \mathrm{cm}^{-1}: 3334,2960,2928,2855,1675$, $1633,1596,1520,1375,1347,1216,1123,1058,1003$, 946, 867, 622. ${ }^{~} \mathrm{H}$ NMR $\left(\mathrm{CDCl}_{3}, 400 \mathrm{MHz}\right) \delta 8.18(\mathrm{~d}, 2 \mathrm{H}$, $J 8.8 \mathrm{~Hz}), 7.47(\mathrm{~d}, 2 \mathrm{H}, J 8.4 \mathrm{~Hz}), 6.53(\mathrm{~s}, 1 \mathrm{H}), 5.03(\mathrm{~s}, 1 \mathrm{H})$, 4.42-4.37 (m, 2H), 2.69-2.65 (m, 2H), 2.27-2.23 (m, 2H), $1.13(\mathrm{~s}, 3 \mathrm{H}), 1.04(\mathrm{~s}, 3 \mathrm{H}) \cdot{ }^{13} \mathrm{C} \mathrm{NMR}\left(\mathrm{CDCl}_{3}, 100 \mathrm{MHz}\right)$ $\delta$ 196.0, 170.8, 167.0, 164.4, 150.0, 147.4, 137.9, 129.1, 124.1, 112.6, 111.5, 60.6, 50.3, 40.9, 38.4, 32.3, 28.9, 27.5. 
MS (ESI): $m / z 398[\mathrm{M}+\mathrm{H}]^{+}$. Anal. calc. for $\mathrm{C}_{21} \mathrm{H}_{19} \mathrm{NO}_{7}$ : C 63.47, H 482, N 3.52; found: C 63.35, H 4.850, N 3.57.

2-(Hydroxymethyl)-7,7-dimethyl-10-(3-nitrophenyl)-7,8dihydropyrano $3,2-b]$ chromene-4,9(6H,10H)-dione $(4 \boldsymbol{e})$

IR (KBr) $v_{\max } / \mathrm{cm}^{-1}: 3393,2955,2922,2851,1669$, 1637, 1599, 1530, 1448, 1377, 1350, 1213, 1143, 1080, 678. ${ }^{1} \mathrm{H} \mathrm{NMR}\left(\mathrm{CDCl}_{3}, 400 \mathrm{MHz}\right) \delta 8.11-8.09(\mathrm{~m}, 2 \mathrm{H}), 7.63$ $(\mathrm{d}, 1 \mathrm{H}, J 8.0 \mathrm{~Hz}), 7.50(\mathrm{t}, 1 \mathrm{H}, J 8.0 \mathrm{~Hz}), 6.53(\mathrm{~s}, 1 \mathrm{H}), 5.03$ (s, 1H), 4.45-4.33 (m, 2H), 274-2.61 (m, 2H), 2.30-2.20 $(\mathrm{m}, 2 \mathrm{H}), 1.12$ (s, 3H), 1.05 (s, 3H). ${ }^{13} \mathrm{C}$ NMR $\left(\mathrm{CDCl}_{3}\right.$, $100 \mathrm{MHz}) \delta 196.3,171.3,168.2,164.7,150.2,148.4,142.4$, 137.7, 134.3, 129.8, 123.4, 122.9, 112.2, 111.3, 60.4, 50.3, 40.8, 38.3, 32.3, 28.9, 27.5. MS (ESI): $\mathrm{m} / \mathrm{z}, 398[\mathrm{M}+\mathrm{H}]^{+}$. Anal. calc. for $\mathrm{C}_{21} \mathrm{H}_{19} \mathrm{NO}_{7}: \mathrm{C} 63.47, \mathrm{H} 482, \mathrm{~N} 3.52$; found: C 63.39, H 4.80, N 3.55.

2-(Hydroxymethyl)-7,7-dimethyl-10-(2-chlorophenyl)-7,8dihydropyrano[3,2-b]chromene-4,9(6H,10H)-dione (4f)

IR $(\mathrm{KBr}) \mathrm{v}_{\max } / \mathrm{cm}^{-1}: 3291,2956,2931,2860,1673$, 1634, 1600, 1468, 1445, 1378, 1221, 1116, 1080, 758. ${ }^{1} \mathrm{H} \mathrm{NMR}\left(\mathrm{CDCl}_{3}, 400 \mathrm{MHz}\right) \delta 7.36(\mathrm{dd}, 1 \mathrm{H}, J 0.8,8.0 \mathrm{~Hz})$, 7.20-7.16 (m, 3H), $6.49(\mathrm{~s}, 1 \mathrm{H}), 5.44(\mathrm{~s}, 1 \mathrm{H}), 4.40-4.34(\mathrm{~m}$, $2 \mathrm{H}), 2.67-2.64(\mathrm{~m}, 2 \mathrm{H}), 2.26-2.22(\mathrm{~m}, 2 \mathrm{H}), 1.12(\mathrm{~s}, 3 \mathrm{H})$, 1.08 (s, 3H). ${ }^{13} \mathrm{C}$ NMR $\left(\mathrm{CDCl}_{3}, 100 \mathrm{MHz}\right) \delta 196.1,171.3$, 168.0, 164.7, 150.9, 137.8, 137.5, 133.7, 130.1, 129.0, 127.3, 112.0, 111.5, 60.4, 50.3, 40.8, 35.7, 32.2, 29.0, 27.5; MS (ESI): $m / z, 387[\mathrm{M}+\mathrm{H}]^{+}$. Anal. calc. for $\mathrm{C}_{21} \mathrm{H}_{19} \mathrm{ClO}_{5}$ : C 65.20, H 4.95; found: C 65.30, H 4.82.

2-(Hydroxymethyl)-7,7-dimethyl-10-(2,4-dichlorophenyl)7,8-dihydropyrano $[3,2-b]$ chromene-4,9(6H,10H)-dione (4g)

IR (KBr) $v_{\text {max }} / \mathrm{cm}^{-1}: 3296,2952,2863,1676,1637$, $1600,1445,1376,1221,1101,1079,854 .{ }^{1} \mathrm{H} \mathrm{NMR}\left(\mathrm{CDCl}_{3}\right.$, $400 \mathrm{MHz}) \delta 7.39(\mathrm{~s}, 1 \mathrm{H}), 7.21-7.18(\mathrm{~m}, 1 \mathrm{H}), 7.14-7.12$ (m, 1H), $6.50(\mathrm{~s}, 1 \mathrm{H}), 5.39(\mathrm{~s}, 1 \mathrm{H}), 4.41-4.37(\mathrm{~m}, 2 \mathrm{H})$, 2.71-2.65 (m, 2H), 2.26-2.22 (m, 2H), $1.13(\mathrm{~s}, 3 \mathrm{H}), 1.08(\mathrm{~s}$, $3 \mathrm{H}) .{ }^{13} \mathrm{C}$ NMR $\left(\mathrm{CDCl}_{3}, 100 \mathrm{MHz}\right) \delta$ 196.0, 171.1, 167.6, $164.8,150.3,137.7,134.4,134.2,130.9,129.9,127.7$, 112.2, 111.2, 60.5, 50.3, 40.8, 37.5, 32.2, 28.9, 27.6. MS (ESI): $m / z 422[\mathrm{M}+\mathrm{H}]^{+}$. Anal. calc. for $\mathrm{C}_{21} \mathrm{H}_{18} \mathrm{Cl}_{2} \mathrm{O}_{5:} \mathrm{C} 59.87$, H 4.31; found: C 59.90, H 4.28.

2-(Hydroxymethyl)-7,7-dimethyl-10-(3,4,5trimethoxyphenyl)-7,8-dihydropyrano [3,2-b]chromene$4,9(6 \mathrm{H}, 10 \mathrm{H})$-dione $(\mathbf{4 h})$

IR (KBr) $v_{\max } / \mathrm{cm}^{-1}: 3296,2960,2923,2869,1673$, 1637, 1598, 1508, 1422, 1375, 1329, 1220, 1126, 1076, 956. ${ }^{1} \mathrm{H}$ NMR $\left(\mathrm{CDCl}_{3}, 400 \mathrm{MHz}\right) \delta 6.50-6.44(\mathrm{~m}, 3 \mathrm{H})$, $4.81(\mathrm{~s}, 1 \mathrm{H}), 4.45-4.37(\mathrm{~m}, 2 \mathrm{H}), 3.80(\mathrm{~s}, 6 \mathrm{H}), 3.79(\mathrm{~s}, 3 \mathrm{H})$, 2.68-2.63 (m, 2H), 2.28-2.27 (m, 2H), $1.14(\mathrm{~s}, 3 \mathrm{H}), 1.11(\mathrm{~s}$, $3 \mathrm{H}) .{ }^{13} \mathrm{C}$ NMR $\left(\mathrm{CDCl}_{3}, 100 \mathrm{MHz}\right) \delta 196.3,171.3,167.7$, 164.0, 153.4, 151.5, 137.3, 136.1, 112.1, 105.0, 60.8, 60.6, 56.1, 50.3, 40.9, 38.5, 32.2, 29.3, 27.1; MS (ESI): $m / z 443$ $[\mathrm{M}+\mathrm{H}]^{+}$. Anal. calc. for $\mathrm{C}_{24} \mathrm{H}_{26} \mathrm{O}_{8}: \mathrm{C} 65.15, \mathrm{H}$ 5.92; found: C 65.20, H 5.89.

2-(Hydroxymethyl)-7,7-dimethyl-10-(2,5-dimethoxyphenyl)-7,8-dihydropyrano[3,2-b]chromene- 4,9(6H, $10 \mathrm{H})$-dione (4i)

IR (KBr) $v_{\max } / \mathrm{cm}^{-1}: 3284,2950,2930,2854,1672,1636$, 1594, 1503, 1449, 1379, 1226, 1194, 1148, 1080, 1047, 819, 709; ${ }^{1} \mathrm{H}$ NMR $\left(\mathrm{CDCl}_{3}, 400 \mathrm{MHz}\right) \delta$ 6.77-6.74 (m, $3 \mathrm{H}), 6.47(\mathrm{~s}, 1 \mathrm{H}), 5.17(\mathrm{~s}, 1 \mathrm{H}), 4.40-4.35(\mathrm{~m}, 2 \mathrm{H}), 3.78(\mathrm{~s}$, $3 \mathrm{H}), 3.72(\mathrm{~s}, 3 \mathrm{H}), 2.63-2.61(\mathrm{~m}, 2 \mathrm{H}), 2.24-2.20(\mathrm{~m}, 2 \mathrm{H})$, $1.11(\mathrm{~s}, 3 \mathrm{H}), 1.04(\mathrm{~s}, 3 \mathrm{H}) ;{ }^{13} \mathrm{C}$ NMR $\left(\mathrm{CDCl}_{3}, 100 \mathrm{MHz}\right)$ $\delta$ 196.3, 171.3, 167.3, 164.6, 153.7, 151.7, 151.6, 137.8, 129.4, 116.1, 113.4, 112.7, 112.1, 111.4, 60.6, 56.6, 55.6, 50.4, 40.9, 33.8, 32.2, 29.2, 27.1; MS (ESI): $\mathrm{m} / \mathrm{z} 413$ $[\mathrm{M}+\mathrm{H}]^{+}$. Anal. calc. for $\mathrm{C}_{23} \mathrm{H}_{24} \mathrm{O}_{7}: \mathrm{C} 66.98, \mathrm{H}$ 5.87; found: C 67.05, H 5.80.

2-(Hydroxymethyl)-7,7-dimethyl-10-(4-methylphenyl)-7,8dihydropyrano[3,2-b]chromene-4,9(6H,10H)-dione (4j)

IR (KBr) $v_{\text {max }} / \mathrm{cm}^{-1}: 3368,2953,2931,2847,1668$, 1636, 1442, 1376, 1219, 1189, 1120, 1076, 950, 862, 624. ${ }^{1} \mathrm{H}$ NMR $\left(\mathrm{CDCl}_{3}, 400 \mathrm{MHz}\right) \delta 7.13(\mathrm{~d}, 2 \mathrm{H}, J 8.0 \mathrm{~Hz}), 7.08$ $(\mathrm{d}, 2 \mathrm{H}, J 8.0 \mathrm{~Hz}), 6.50(\mathrm{~s}, 1 \mathrm{H}), 4.83(\mathrm{~s}, 1 \mathrm{H}), 4.38-4.34(\mathrm{~m}$, $2 \mathrm{H}), 2.64-2.61(\mathrm{~m}, 2 \mathrm{H}), 2.28(\mathrm{~s}, 3 \mathrm{H}), 2.23-2.20(\mathrm{~m}, 2 \mathrm{H})$, $1.10(\mathrm{~s}, 3 \mathrm{H}), 1.03(\mathrm{~s}, 3 \mathrm{H}) ;{ }^{13} \mathrm{C}$ NMR $\left(\mathrm{CDCl}_{3}, 100 \mathrm{MHz}\right)$ $\delta$ 196.4, 171.5, 168.2, 163.9, 152.0, 137.6, 137.5, 137.3, 129.5, 127.9, 112.4, 111.9, 60.4, 50.4, 40.8, 37.9, 32.2, 29.0, 27.4, 21.1; MS (ESI): $\mathrm{m} / z 367[\mathrm{M}+\mathrm{H}]^{+}$. Anal. calc. for $\mathrm{C}_{22} \mathrm{H}_{22} \mathrm{O}_{5}: \mathrm{C}$ 72.12, $\mathrm{H}$ 6.05; found: C 72.20, H 6.00.

2-(Hydroxymethyl)-7,7-dimethyl-10-(4-methoxyphenyl)7,8-dihydropyrano [3,2-b]chromene-4,9(6H,10H)-dione (4k)

IR (KBr) $v_{\text {max }} / \mathrm{cm}^{-1}: 3355,2958,2922,2836,1672,1637$, 1511, 1443, 1377, 1219, 1193, 1120, 1029, 950, 860, 629; ${ }^{1} \mathrm{H} \mathrm{NMR}\left(\mathrm{CDCl}_{3}, 400 \mathrm{MHz}\right) \delta$ 7.18-7.16 (m, 2H), 6.83-6.80 $(\mathrm{m}, 2 \mathrm{H}), 6.50(\mathrm{~s}, 1 \mathrm{H}), 4.83(\mathrm{~s}, 1 \mathrm{H}), 4.40-4.35(\mathrm{~m}, 2 \mathrm{H}), 3.75$ (s, 3H), 2.65-2.61 (m, 2H), 2.23-2.22 (m, 2H), $1.10(\mathrm{~s}, 3 \mathrm{H})$, 1.04 (s, 3H); ${ }^{13} \mathrm{C}$ NMR $\left(\mathrm{CDCl}_{3}, 100 \mathrm{MHz}\right) \delta 196.4,171.5$, 168.0, 16.7, 159.0, 152.0, 137.3, 132.7, 129.1, 114.2, 112.5, 129.1, 60.5, 55.2, 50.4, 40.8, 37.5, 32.2, 29.0, 27.4; MS (ESI): $m / z, 383[\mathrm{M}+\mathrm{H}]^{+}$. Anal. calc. for $\mathrm{C}_{22} \mathrm{H}_{22} \mathrm{O}_{6}: \mathrm{C}$ 69.10, H 5.80; found: C 69.20, H5.72. 
(A)

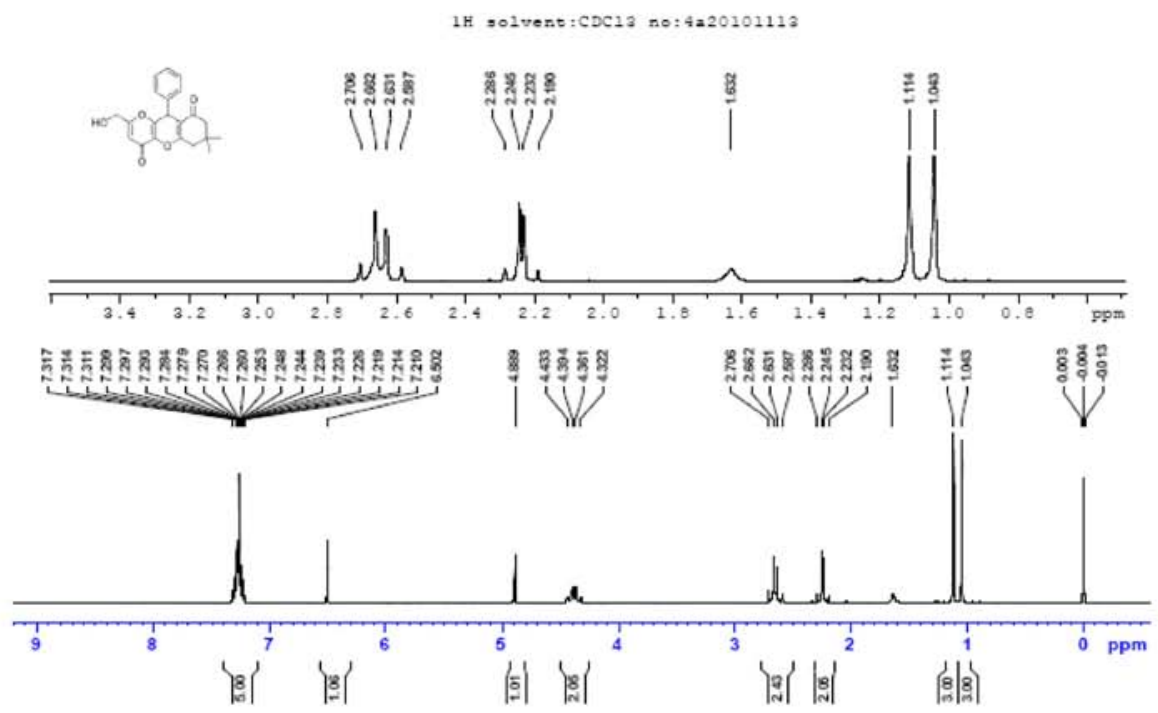

(B)
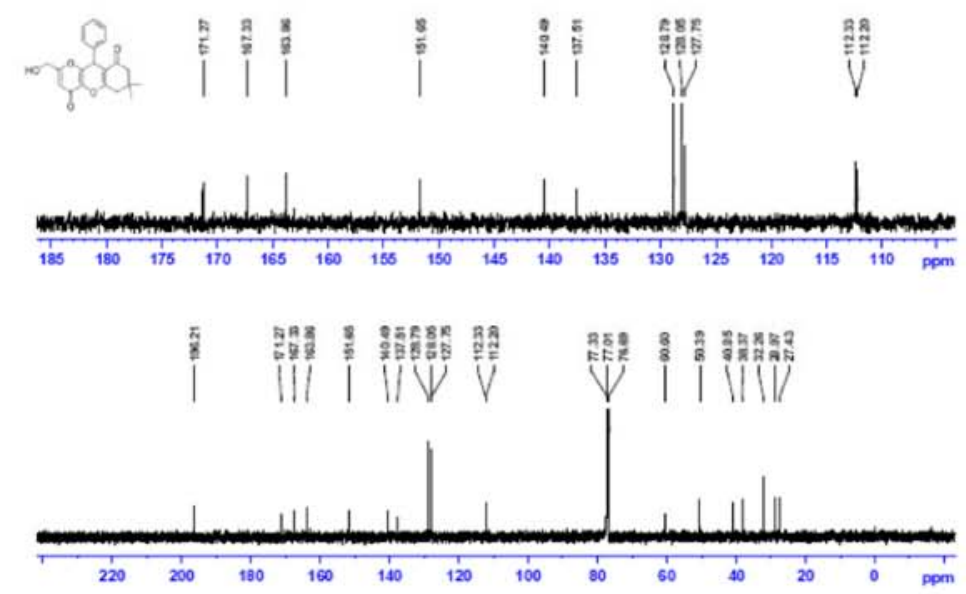

(C)

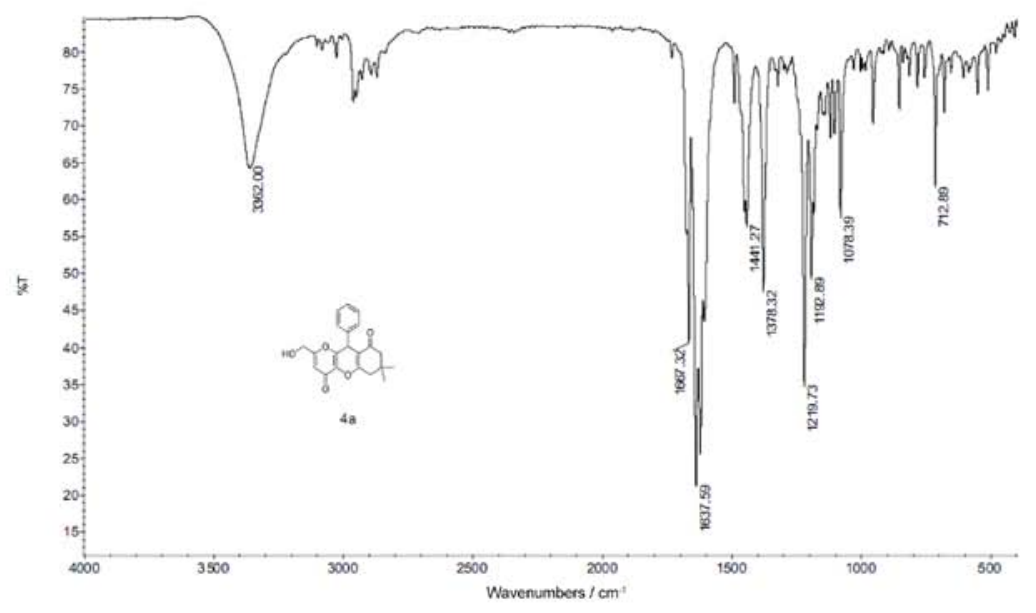

Figure S1. (A) ${ }^{1} \mathrm{H} N M R$ of $\mathbf{4 a}\left(400 \mathrm{MHz}, \mathrm{CDCl}_{3}\right)$. (B) ${ }^{13} \mathrm{C} \mathrm{NMR}$ of $\mathbf{4 a}\left(100 \mathrm{MHz}, \mathrm{CDCl}_{3}\right)$. (C) IR of $\mathbf{4 a}$. 
(A)

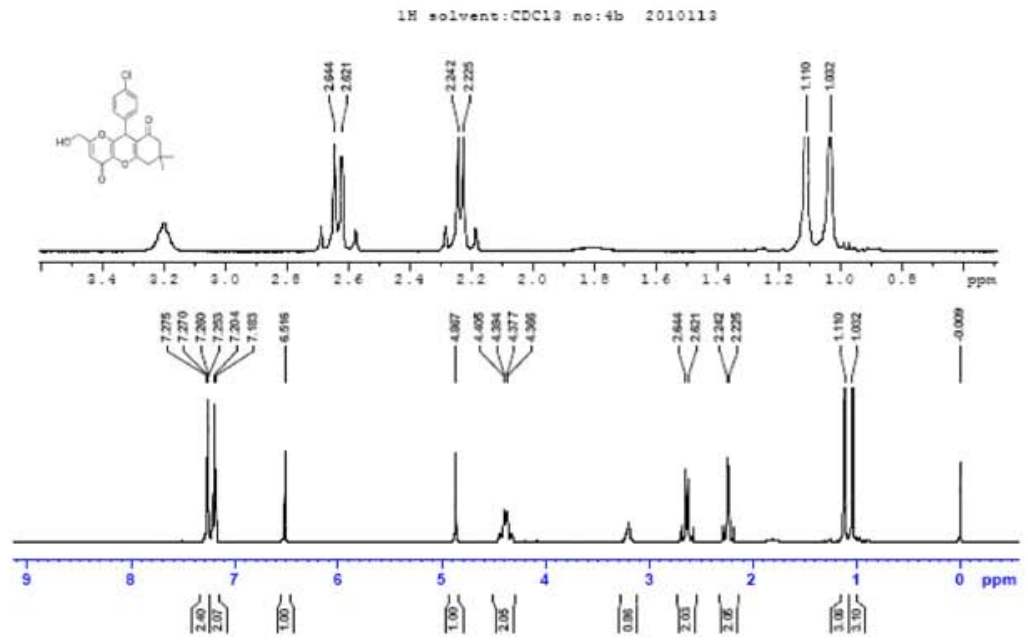

(B)
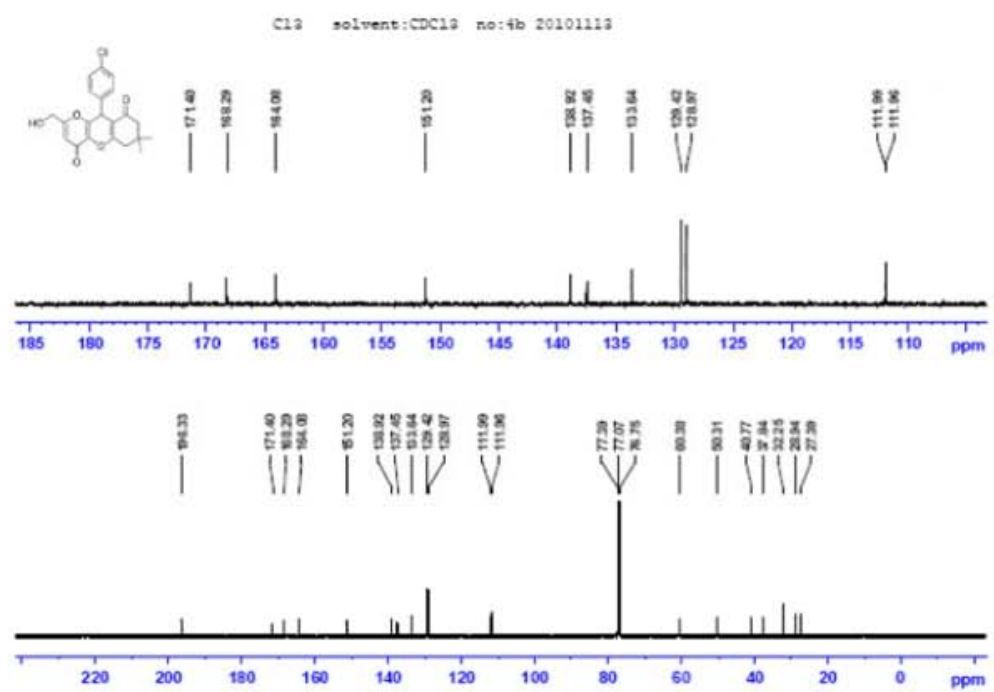

(C)

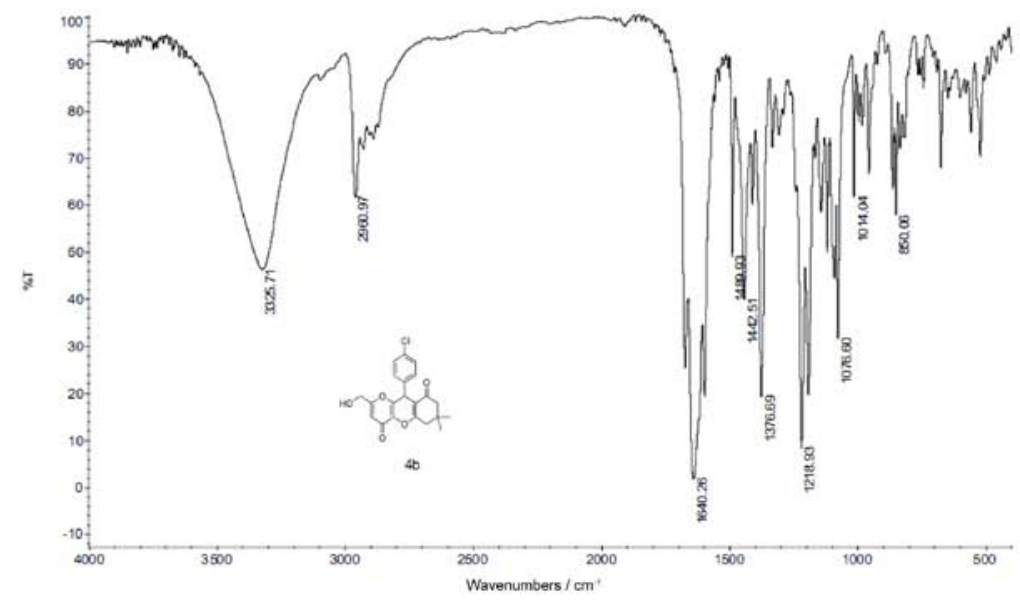

Figure S2. (A) ${ }^{1} \mathrm{H}$ NMR of $\mathbf{4 b}\left(400 \mathrm{MHz}, \mathrm{CDCl}_{3}\right)$. (B) ${ }^{13} \mathrm{C}$ NMR of $\mathbf{4 b}\left(100 \mathrm{MHz}, \mathrm{CDCl}_{3}\right)$. (C) IR of $\mathbf{4 b}$. 
(A)

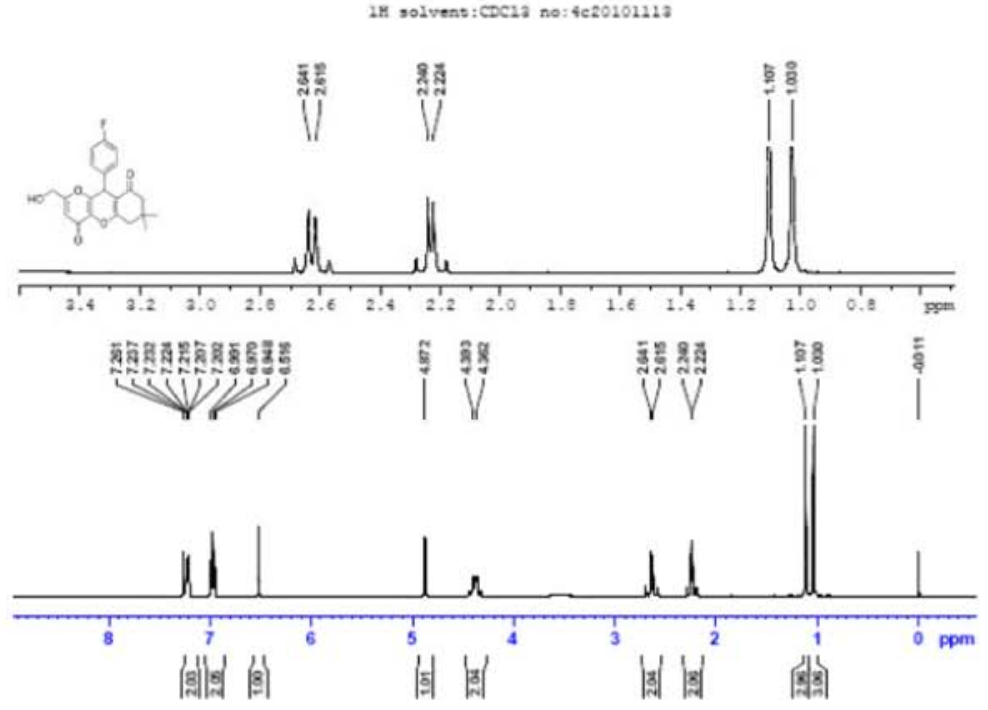

(B)
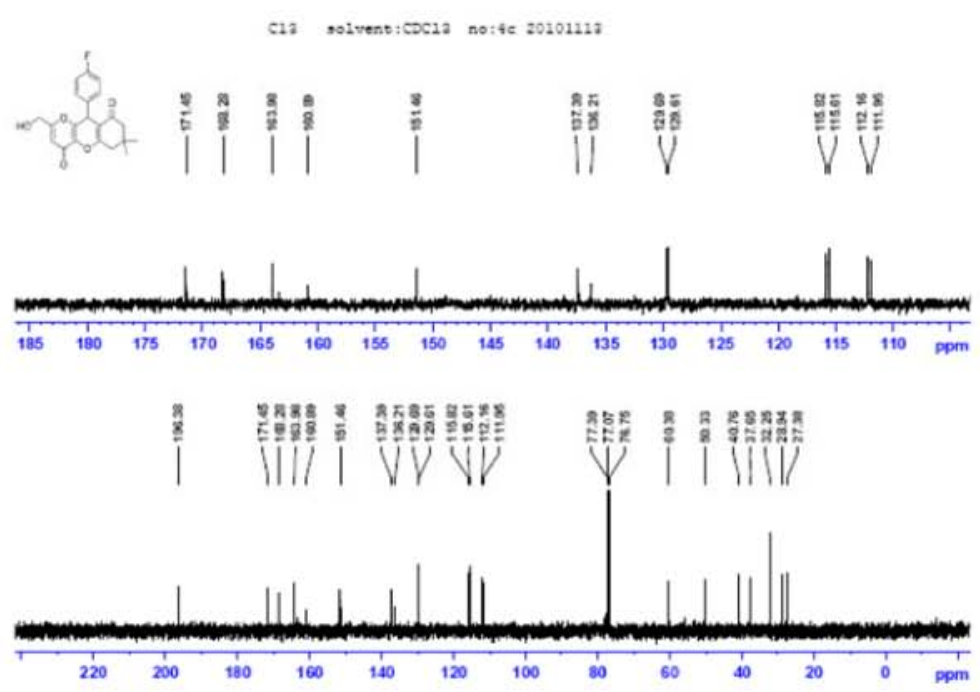

(C)

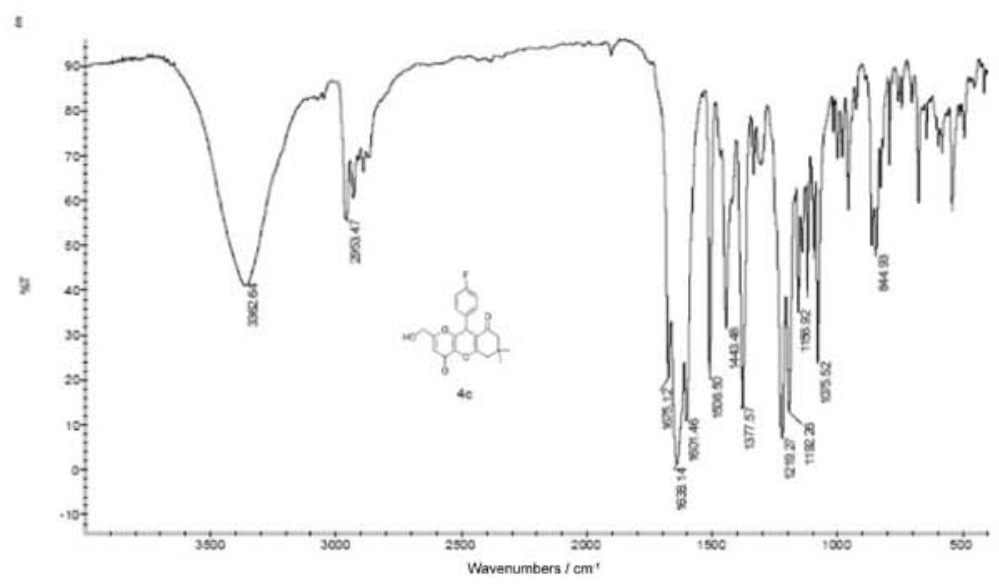

Figure S3. (A) ${ }^{1} \mathrm{H}$ NMR of $4 \mathbf{c}\left(400 \mathrm{MHz}, \mathrm{CDCl}_{3}\right)$. (B) ${ }^{13} \mathrm{C} \mathrm{NMR}$ of $4 \mathbf{c}\left(100 \mathrm{MHz}, \mathrm{CDCl}_{3}\right)$. (C) IR of $\mathbf{4 c}$. 
(A)

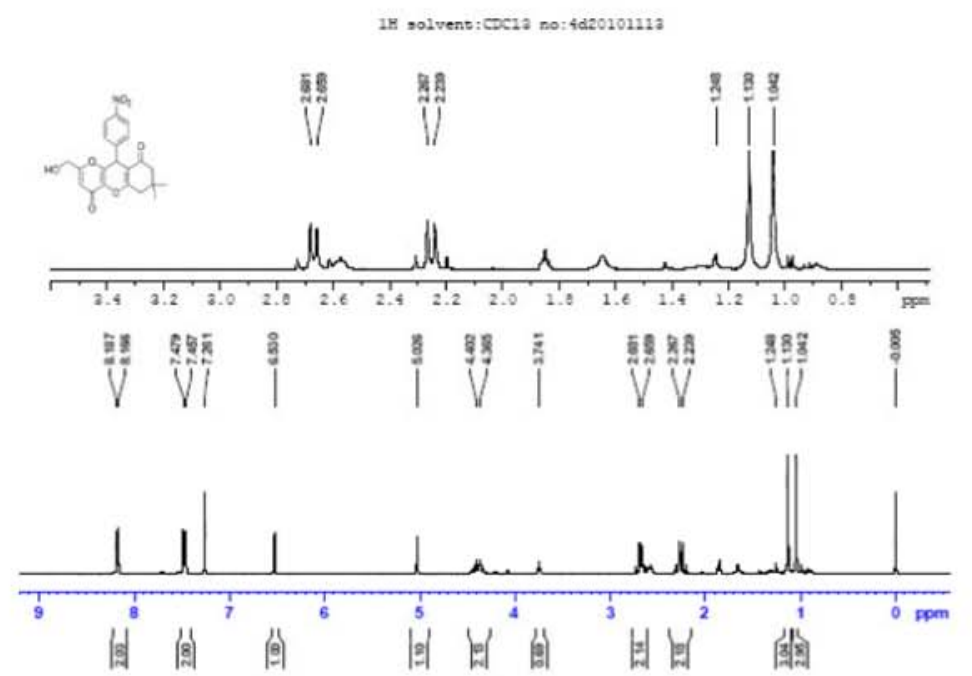

(B)

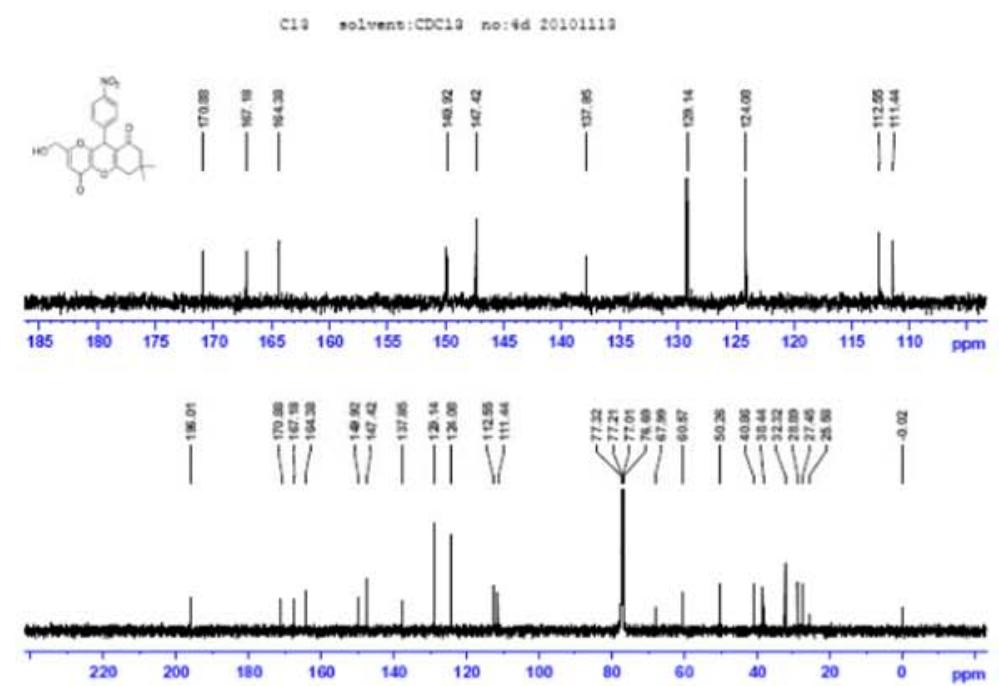

(C)

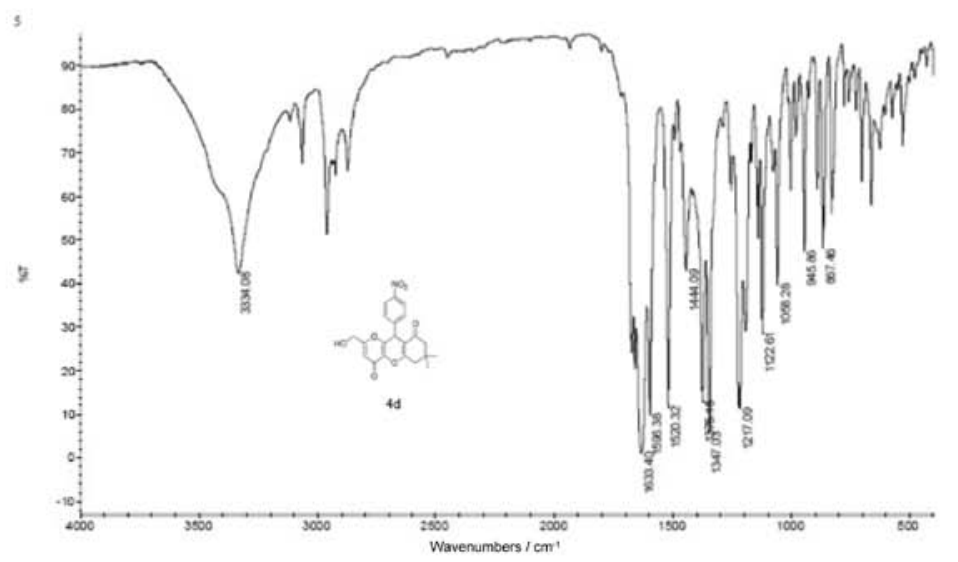

Figure S4. (A) ${ }^{1} \mathrm{H}$ NMR of $4 \mathbf{d}\left(400 \mathrm{MHz}, \mathrm{CDCl}_{3}\right)$. (B) ${ }^{13} \mathrm{C} \mathrm{NMR}$ of $4 \mathbf{d}\left(100 \mathrm{MHz}, \mathrm{CDCl}_{3}\right)$. (C) IR of $\mathbf{4 d}$. 
(A)

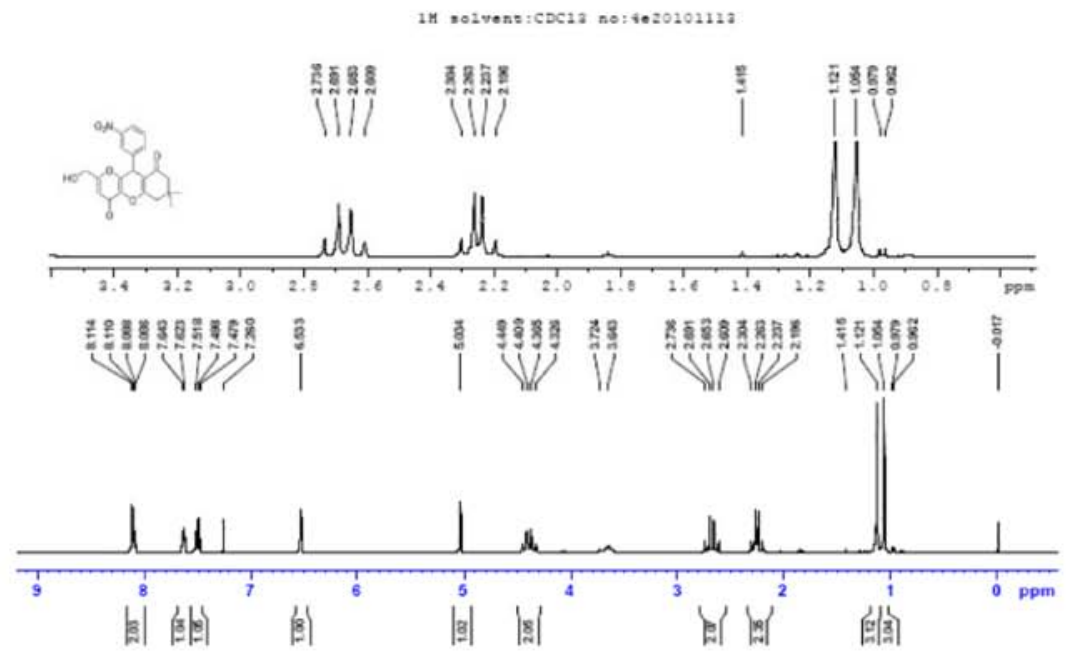

(B)
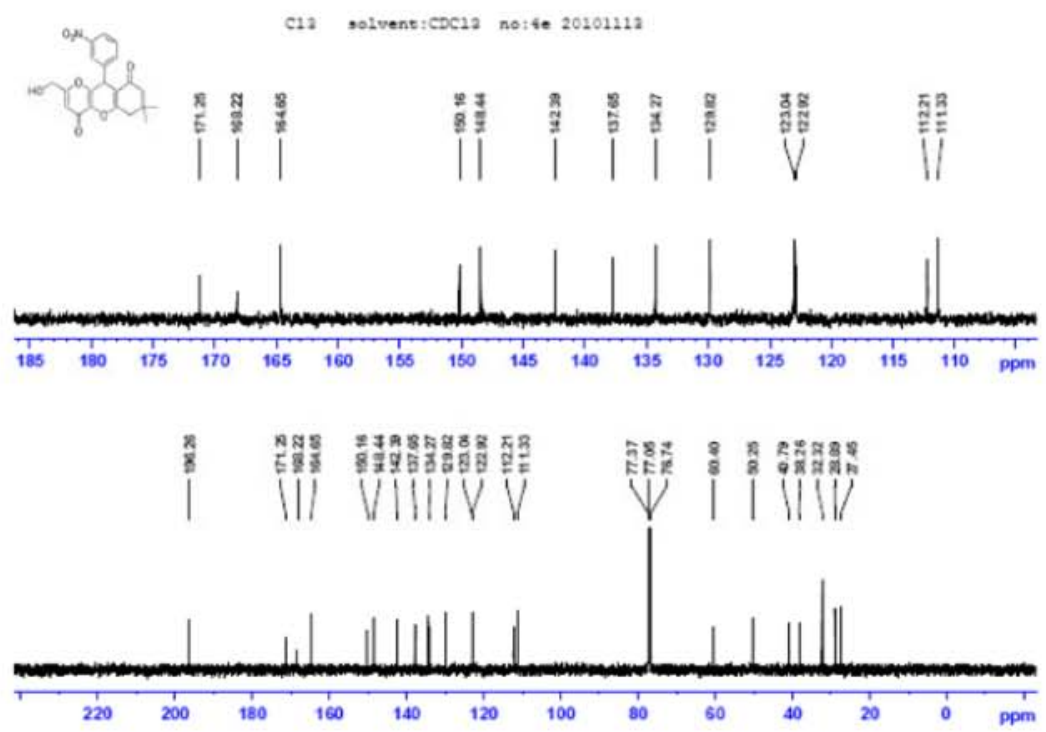

(C)

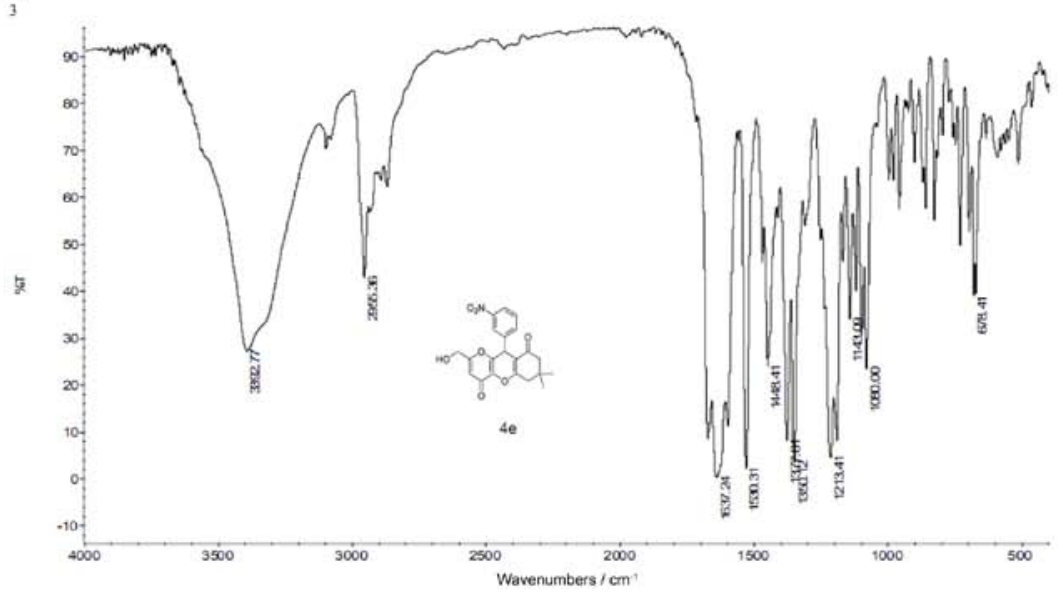

Figure S5. (A) ${ }^{1} \mathrm{H} N M R$ of $4 \mathbf{e}\left(400 \mathrm{MHz}, \mathrm{CDCl}_{3}\right)$. (B) ${ }^{13} \mathrm{C} \mathrm{NMR}$ of $4 \mathbf{e}\left(100 \mathrm{MHz}, \mathrm{CDCl}_{3}\right)$. (C) IR of $4 \mathbf{e}$. 
(A)

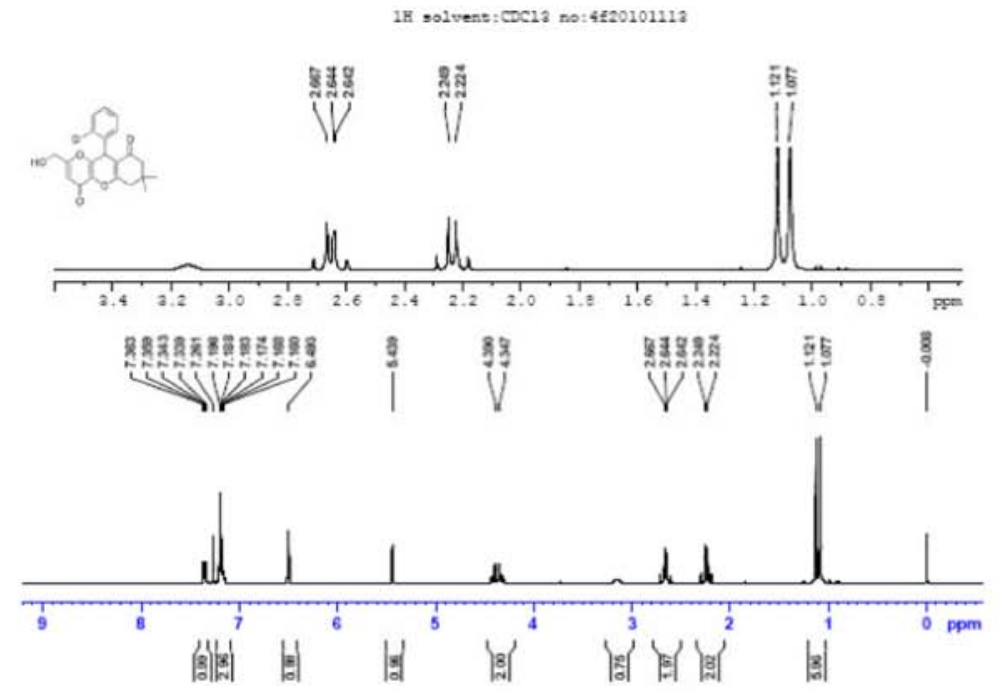

(B)

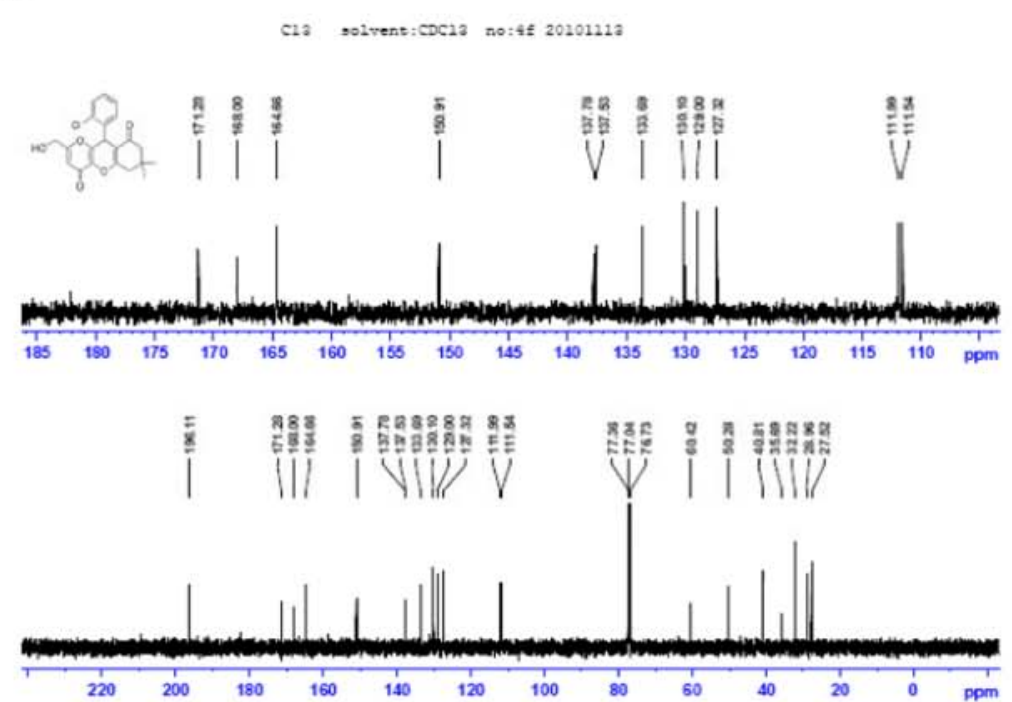

(C)

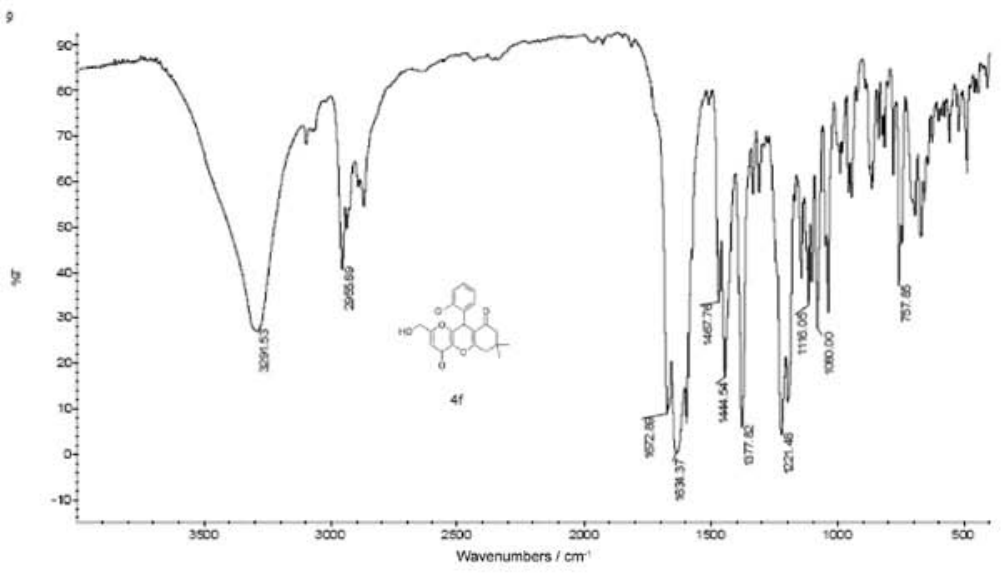

Figure S6. (A) ${ }^{1} \mathrm{H}$ NMR of $\mathbf{4 f}\left(400 \mathrm{MHz}, \mathrm{CDCl}_{3}\right)$. (B) ${ }^{13} \mathrm{C}$ NMR of $\mathbf{4 f}\left(100 \mathrm{MHz}, \mathrm{CDCl}_{3}\right)$. (C) IR of $\mathbf{4 f}$. 
(A)

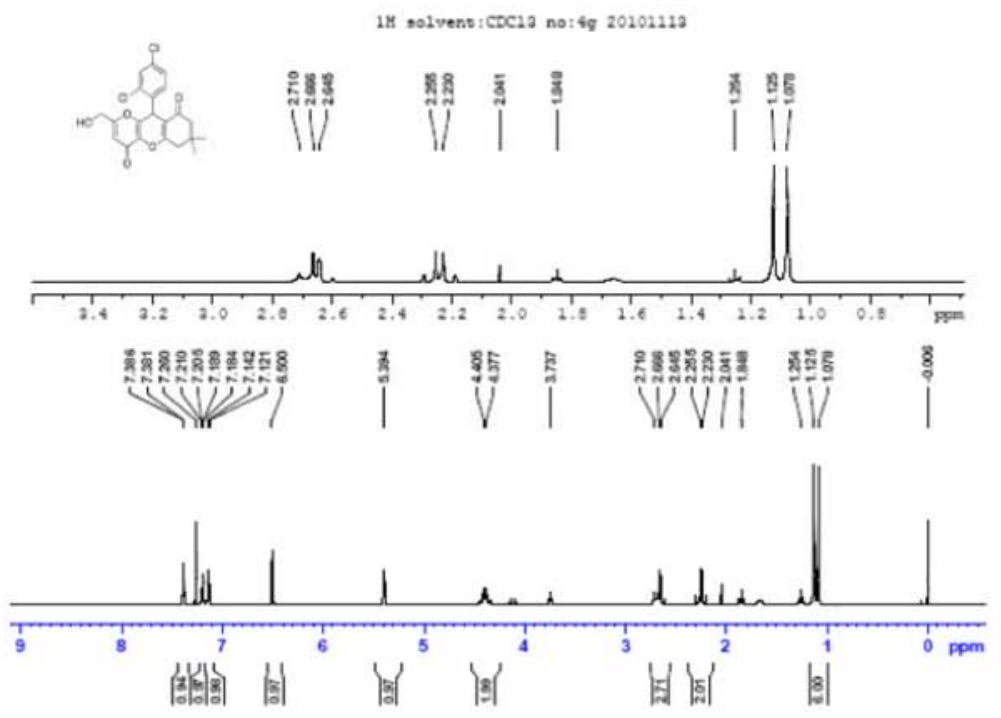

(B)
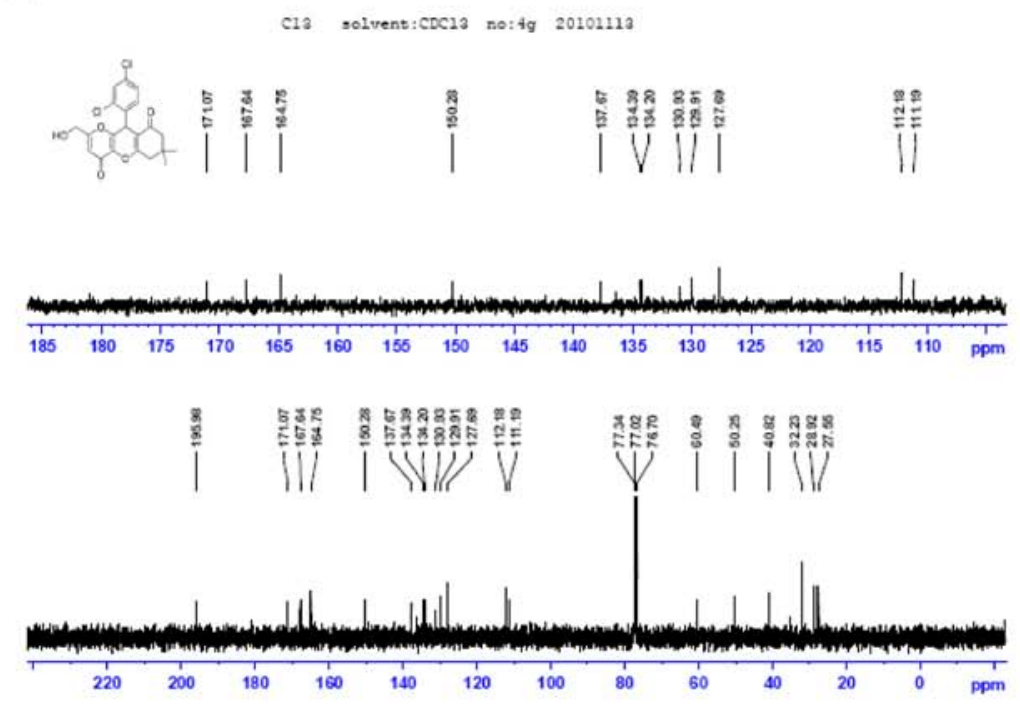

(C)

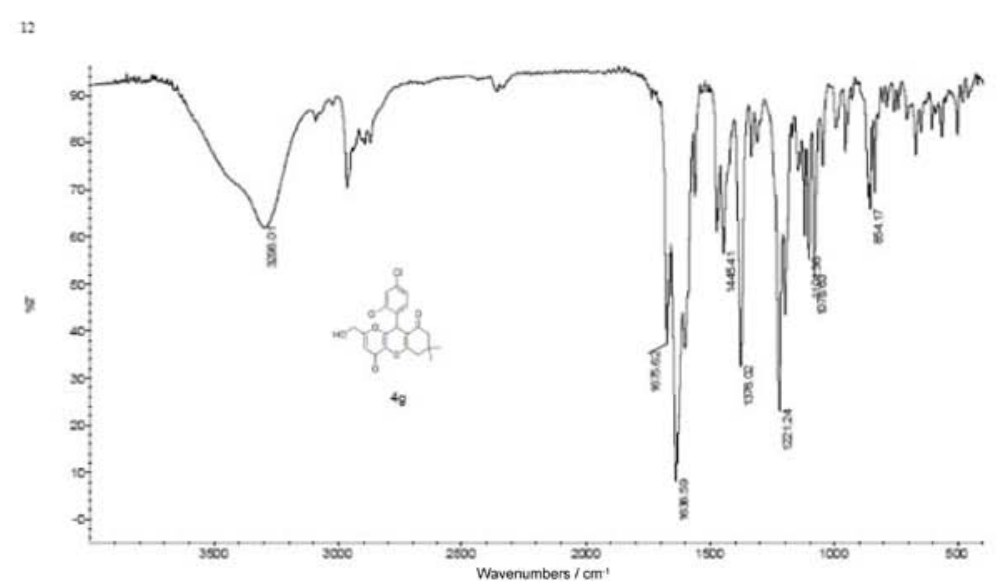

Figure S7. (A) ${ }^{1} \mathrm{H} N M R$ of $\mathbf{4 g}\left(400 \mathrm{MHz}, \mathrm{CDCl}_{3}\right)$. (B) ${ }^{13} \mathrm{C} \mathrm{NMR}$ of $\mathbf{4 g}\left(100 \mathrm{MHz}, \mathrm{CDCl}_{3}\right)$. (C) IR of $\mathbf{4 g}$. 
(A)

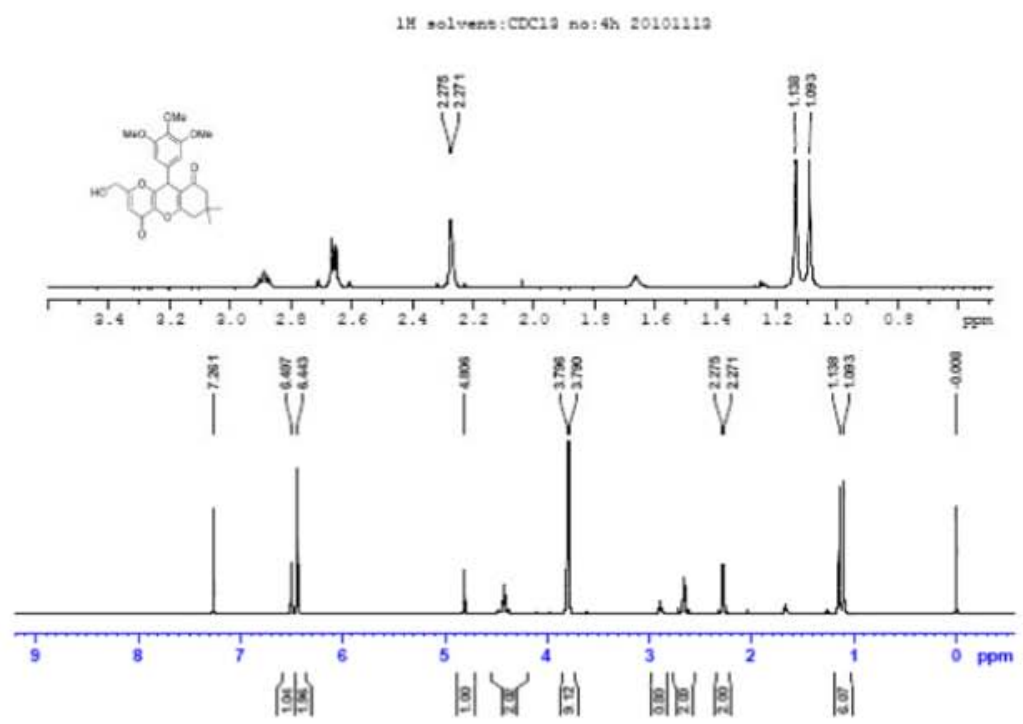

(B)
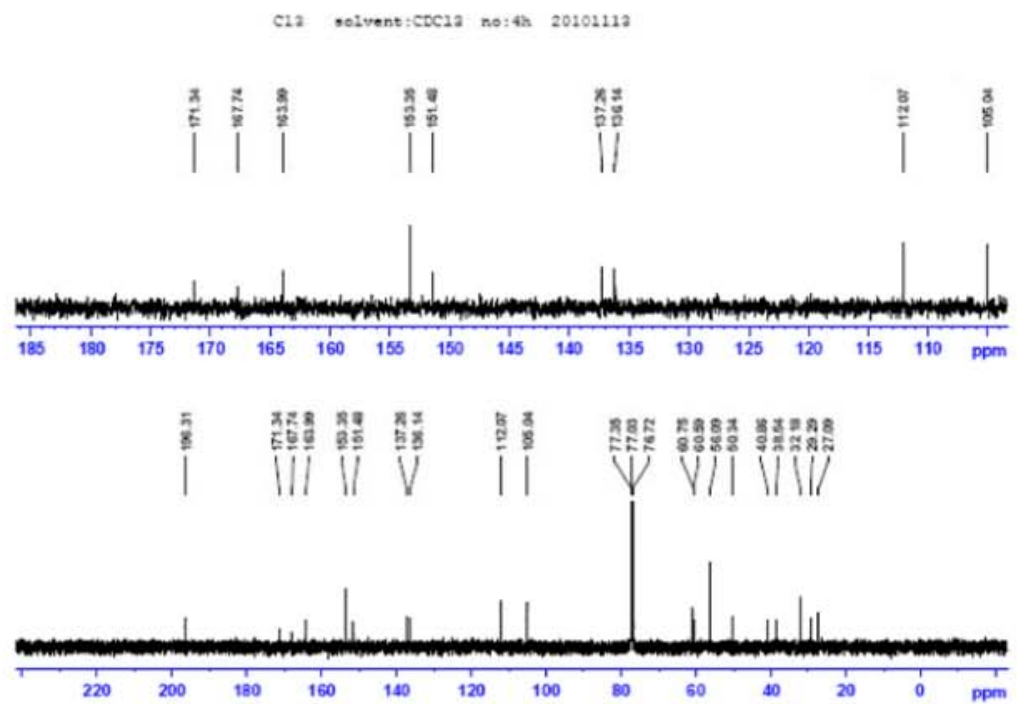

(C)

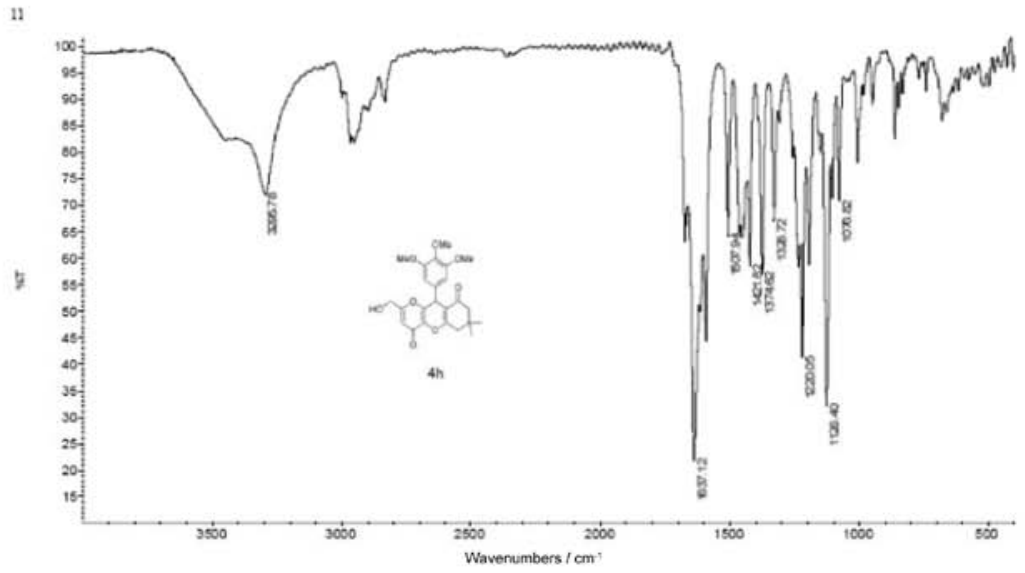

Figure S8. (A) ${ }^{1} \mathrm{H}$ NMR of $4 \mathbf{h}\left(400 \mathrm{MHz}, \mathrm{CDCl}_{3}\right)$. (B) ${ }^{13} \mathrm{C}$ NMR of $4 \mathbf{h}\left(100 \mathrm{MHz}, \mathrm{CDCl}_{3}\right)$. (C) IR of $\mathbf{4 h}$. 
(A)

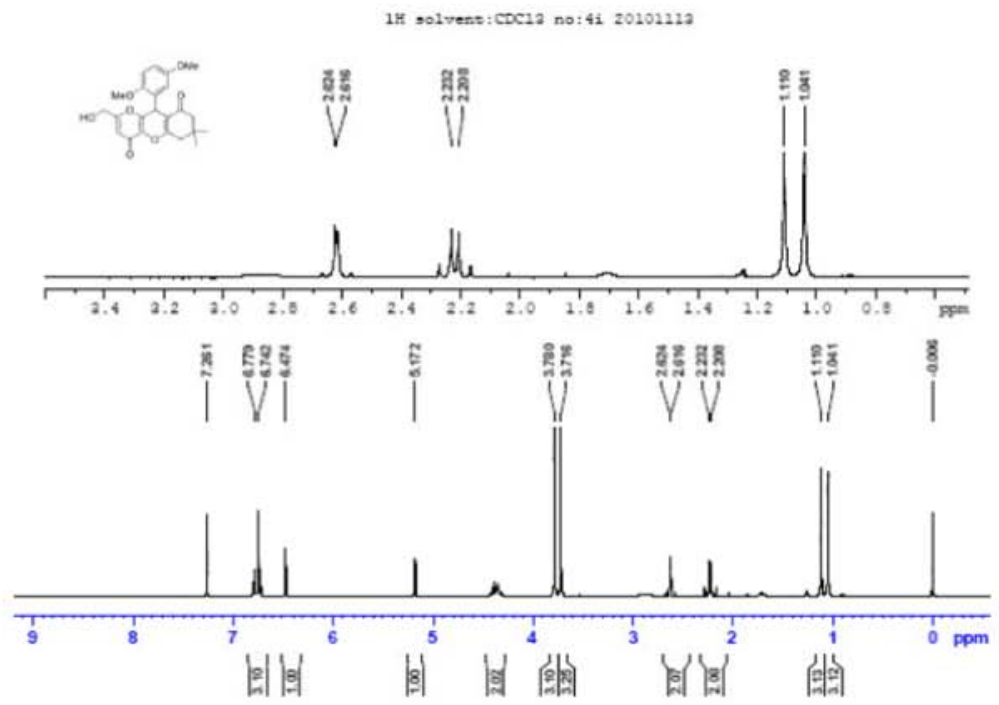

(B)
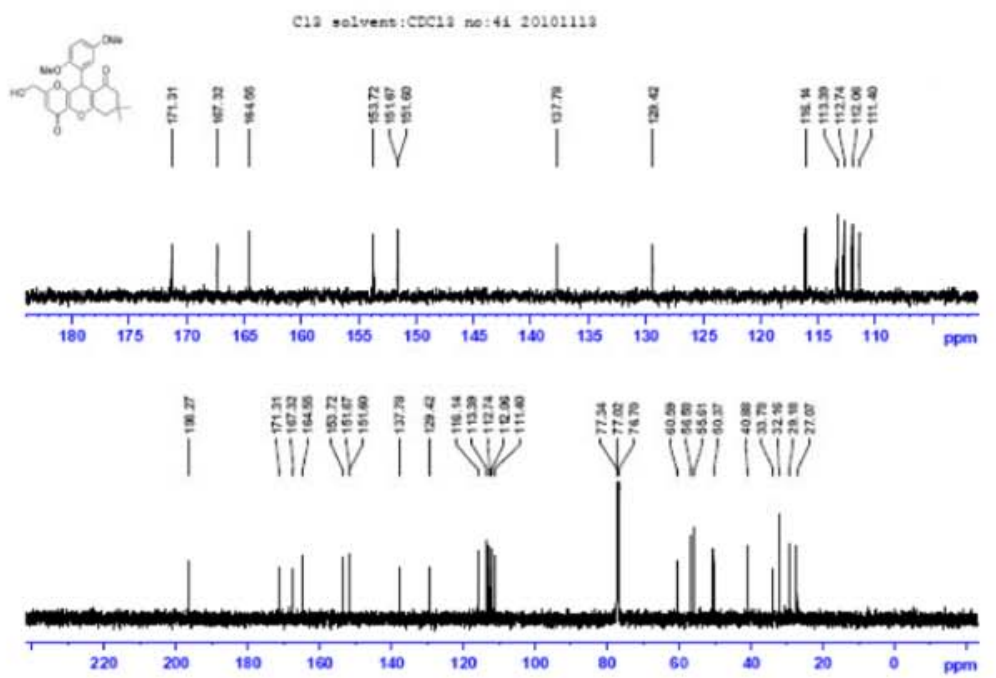

(C)

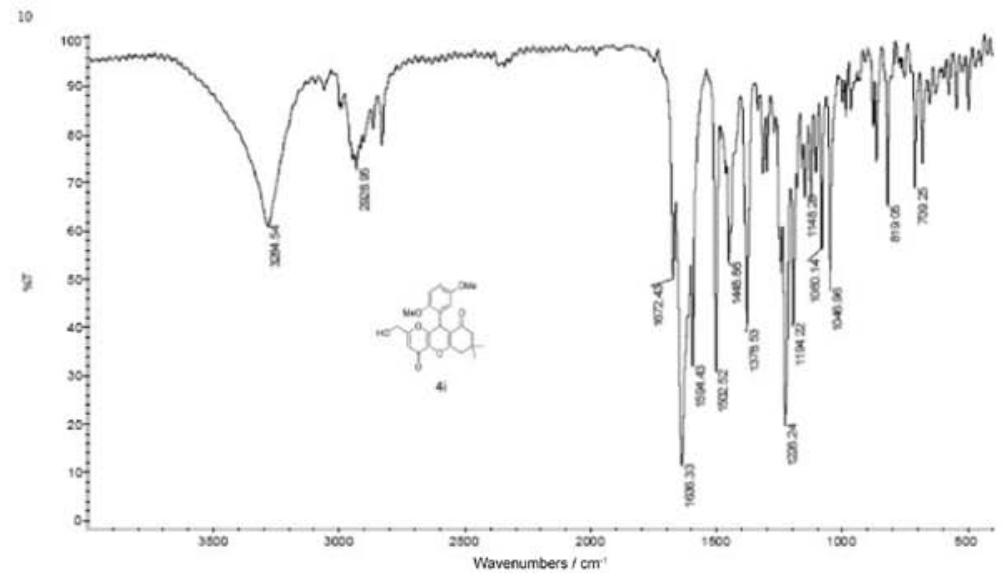

Figure S9. (A) ${ }^{1} \mathrm{H}$ NMR of $4 \mathbf{i}\left(400 \mathrm{MHz}, \mathrm{CDCl}_{3}\right)$. (B) ${ }^{13} \mathrm{C} \mathrm{NMR}$ of $4 \mathbf{i}\left(100 \mathrm{MHz}, \mathrm{CDCl}_{3}\right)$. (C) IR of $4 \mathbf{i}$. 
(A)

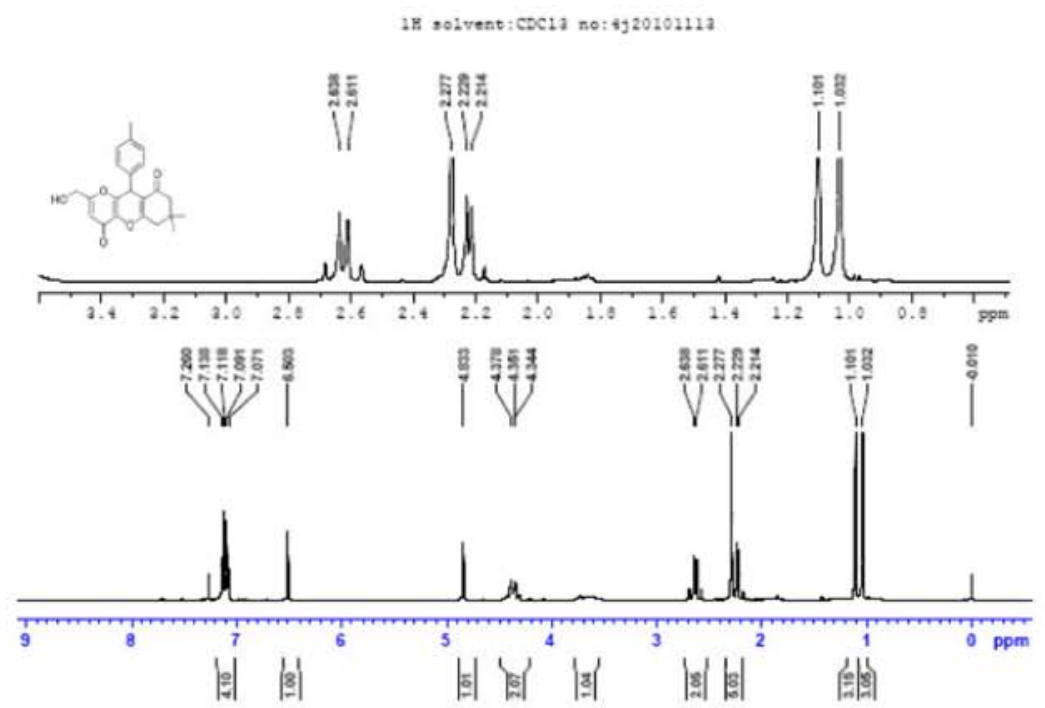

(B)

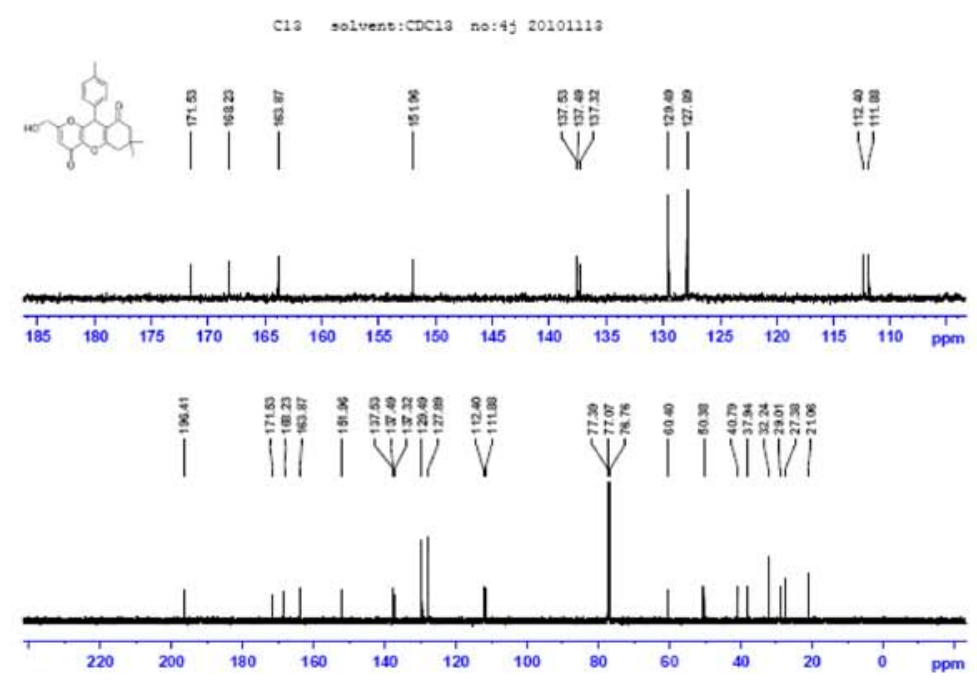

(C)

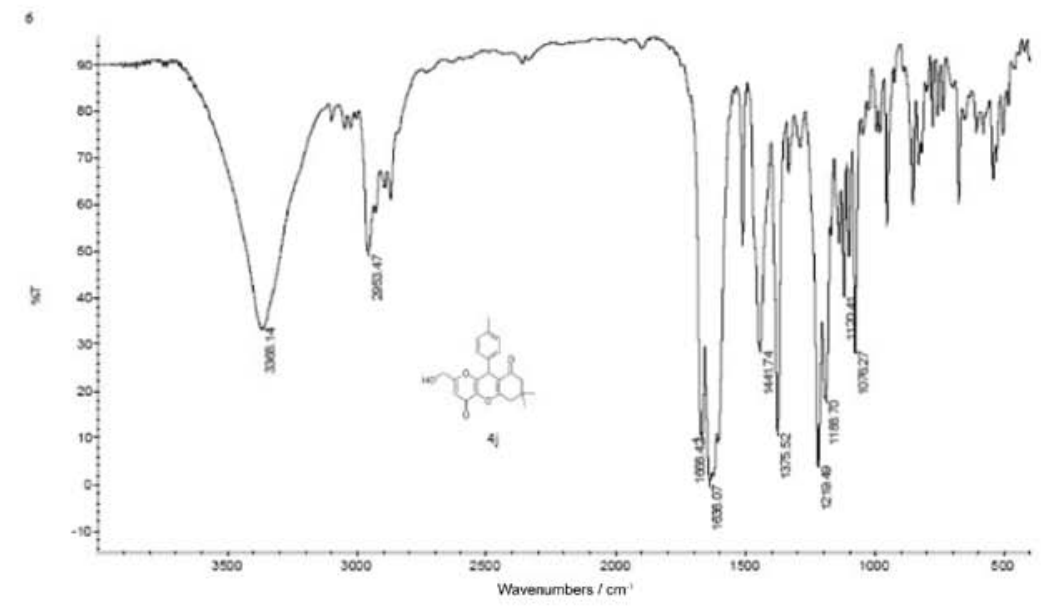

Figure S10. (A) ${ }^{1} \mathrm{H}$ NMR of $\mathbf{4 j}\left(400 \mathrm{MHz}, \mathrm{CDCl}_{3}\right)$. (B) ${ }^{13} \mathrm{C}$ NMR of $\mathbf{4 j}\left(100 \mathrm{MHz}, \mathrm{CDCl}_{3}\right)$. (C) IR of $\mathbf{4 j}$. 
(A)

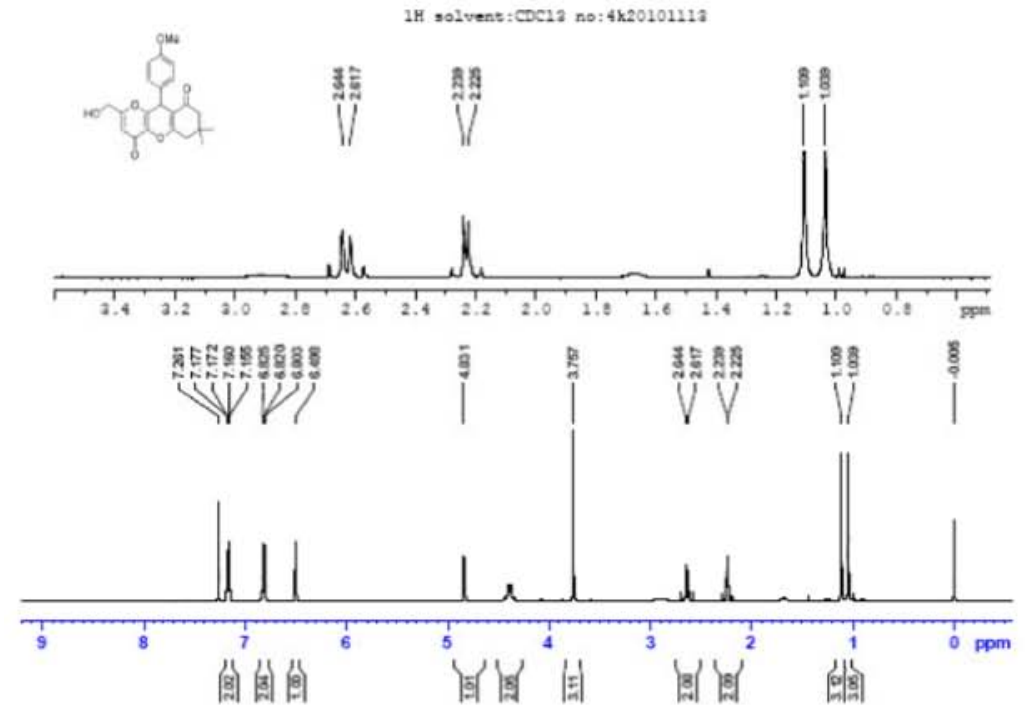

(B)
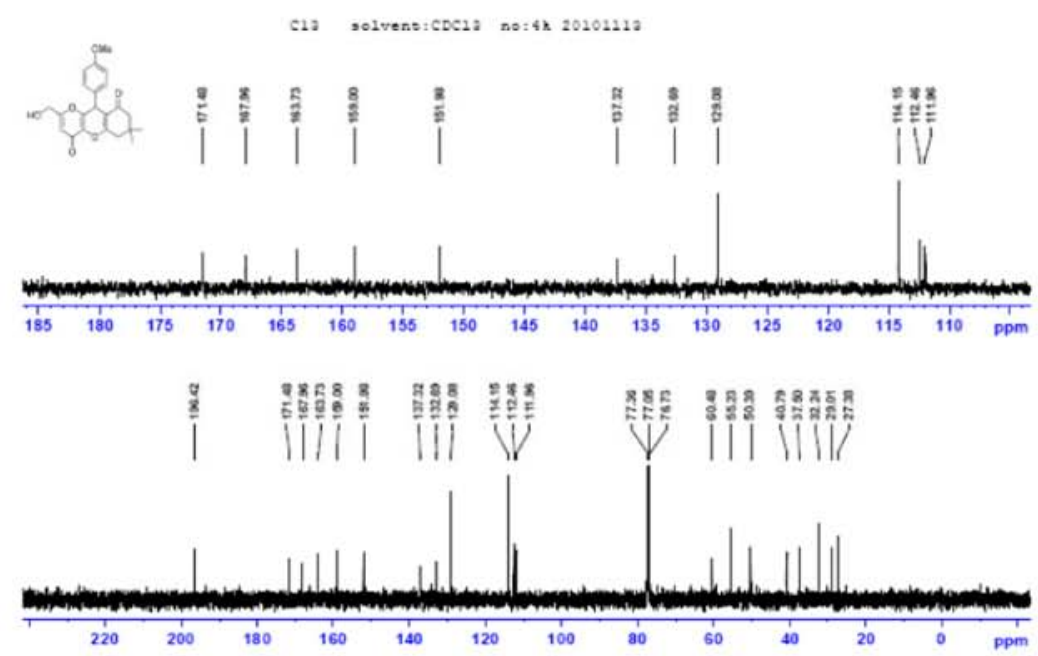

(C)

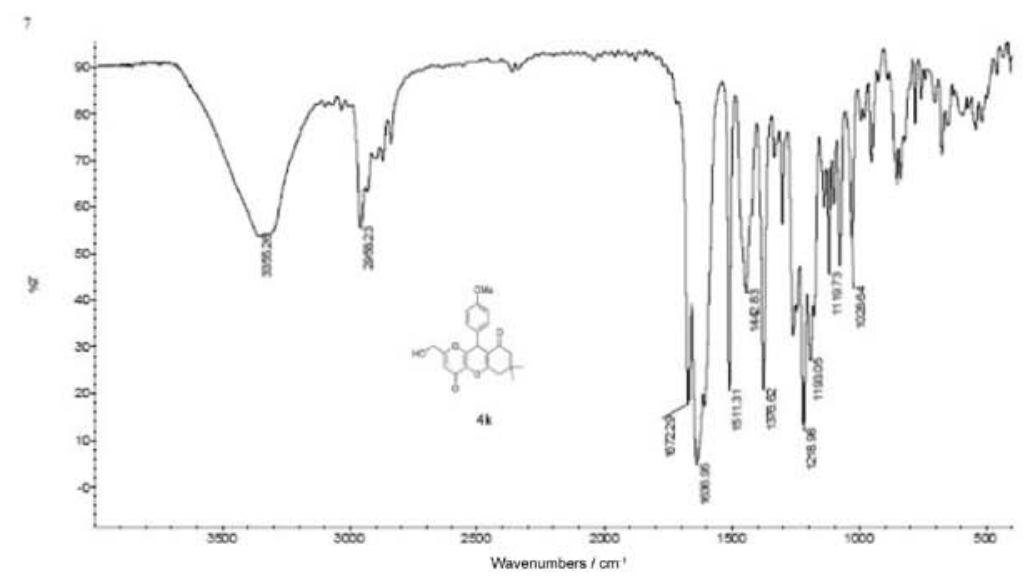

Figure S11. (A) ${ }^{1} \mathrm{H}$ NMR of $\mathbf{4 k}\left(400 \mathrm{MHz}, \mathrm{CDCl}_{3}\right)$. (B) ${ }^{13} \mathrm{C}$ NMR of $\mathbf{4 k}\left(100 \mathrm{MHz}, \mathrm{CDCl}_{3}\right)$. (C) IR of $\mathbf{4 k}$. 